\title{
GENERALITY OF LEAF TRAIT RELATIONSHIPS: A TEST ACROSS SIX BIOMES
}

\author{
Peter B. Reich, ${ }^{1}$ David S. Ellsworth, ${ }^{2}$ Michael B. Walters, ${ }^{3}$ James M. Vose, ${ }^{4}$ Charles Gresham, ${ }^{5}$ \\ John C. Volin, ${ }^{6}$ ANd William D. Bowman ${ }^{7}$ \\ ${ }^{1}$ Department of Forest Resources, University of Minnesota, Saint Paul, Minnesota 55108 USA \\ ${ }^{2}$ Department of Applied Science, Brookhaven National Laboratory, Upton, New York 11973 USA \\ ${ }^{3}$ Department of Forestry, Michigan State University, East Lansing, Michigan 48824 USA \\ ${ }^{4}$ U.S. Forest Service, Coweeta Hydrological Laboratory, Otto, North Carolina 28763 USA \\ ${ }^{5}$ Baruch Forest Institute, Clemson University, Georgetown, South Carolina 29442 USA \\ ${ }^{6}$ Division of Science, Florida Atlantic University, Davie, Florida 33314 USA \\ ${ }^{7}$ Mountain Research Station, Institute of Arctic and Alpine Research, and \\ Department of Evolutionary, Population, and Organismic Biology, University of Colorado, \\ Boulder, Colorado 80309 USA
}

\begin{abstract}
Convergence in interspecific leaf trait relationships across diverse taxonomic groups and biomes would have important evolutionary and ecological implications. Such convergence has been hypothesized to result from trade-offs that limit the combination of plant traits for any species. Here we address this issue by testing for biome differences in the slope and intercept of interspecific relationships among leaf traits: longevity, net photosynthetic capacity $\left(A_{\max }\right)$, leaf diffusive conductance $\left(G_{s}\right)$, specific leaf area (SLA), and nitrogen $(\mathrm{N})$ status, for more than 100 species in six distinct biomes of the Americas. The six biomes were: alpine tundra-subalpine forest ecotone, cold temperate forest-prairie ecotone, montane cool temperate forest, desert shrubland, subtropical forest, and tropical rain forest. Despite large differences in climate and evolutionary history, in all biomes mass-based leaf $\mathrm{N}\left(N_{\text {mass }}\right)$, SLA, $G_{s}$, and $A_{\max }$ were positively related to one another and decreased with increasing leaf life span. The relationships between pairs of leaf traits exhibited similar slopes among biomes, suggesting a predictable set of scaling relationships among key leaf morphological, chemical, and metabolic traits that are replicated globally among terrestrial ecosystems regardless of biome or vegetation type. However, the intercept (i.e., the overall elevation of regression lines) of relationships between pairs of leaf traits usually differed among biomes. With increasing aridity across sites, species had greater $A_{\max }$ for a given level of $G_{s}$ and lower SLA for any given leaf life span. Using principal components analysis, most variation among species was explained by an axis related to mass-based leaf traits $\left(A_{\max }, \mathrm{N}\right.$, and SLA) while a second axis reflected climate, $G_{s}$, and other area-based leaf traits.
\end{abstract}

Key words: alpine; desert; leaf life span; leaf diffusive conductance; nitrogen; photosynthesis; scaling; specific leaf area; temperate forest; tropical forest.

\section{INTRODUCTION}

The idea that disparate plant species from different biomes may share similar leaf form and function has a long history in ecology, but has rarely been tested in a quantitative manner (Beadle 1966, Chabot and Hicks 1982). It is clear that leaf life span, specific leaf area (SLA), nitrogen (N) concentration, leaf diffusive conductance $\left(G_{s}\right)$, and photosynthetic rate are fundamental plant traits that vary greatly among species, often by orders of magnitude (Field and Mooney 1986, Reich et al. 1992). This variation may represent adaptation to environmental heterogeneity that exists both locally and globally. It is well established that these leaf traits are generally correlated, based on examination of variation among and within species (e.g., Lugg and Sinclair 1981,

Manuscript received 26 January 1998; revised 28 August 1998; accepted 7 August 1998.
Field and Mooney 1986, Körner 1989, Reich et al. 1992), but a comprehensive, quantitative evaluation of these relationships among biomes is lacking. If combinations of leaf traits recur in distantly related taxa across a wide biogeographic range it would suggest that selection constrains the collective leaf traits possessed by every species (Reich et al. 1992, Reich 1993) and provide evidence for convergent evolution.

Studies from a variety of species and ecosystems, among plant groups taxonomically either broad (Field and Mooney 1986, Evans 1989, Reich et al. 1991, 1992, Reich 1993, Abrams et al. 1994, Mulkey et al. 1995) or narrow (Chazdon and Field 1987, Williams et al. 1989) have generally reported similar patterns of relationships among leaf traits. Mass-based photosynthetic capacity $\left(A_{\text {mass }}\right)$ and leaf nitrogen $\left(N_{\text {mass }}\right)$ are usually positively correlated, both are positively correlated with SLA, and all three traits decline with increasing 
TABLE 1. Description of the six study sites and related ecosystems used in the study of leaf trait relationships in the northernhemisphere Americas.

\begin{tabular}{|c|c|c|c|c|c|c|}
\hline Site & Location & Ecosystem type & $\begin{array}{l}\text { Elevation } \\
\quad(\mathrm{m})\end{array}$ & $\begin{array}{c}\text { Temp. } \\
\left({ }^{\circ} \mathrm{C}\right)\end{array}$ & $\begin{array}{c}\text { Precip. } \\
(\mathrm{mm})\end{array}$ & $\begin{array}{l}\text { PET } \\
(\mathrm{mm})\end{array}$ \\
\hline \multirow[t]{2}{*}{$\begin{array}{l}\text { Niwot Ridge, Colorado, } \\
\text { USA }\end{array}$} & $\begin{array}{c}40^{\circ} 03^{\prime} \mathrm{N}, 105^{\circ} 36^{\prime} \\
\mathrm{W}\end{array}$ & Alpine tundra & 3510 & \multirow[t]{2}{*}{-3.0} & \multirow[t]{2}{*}{900} & \multirow[t]{2}{*}{$350-400$} \\
\hline & $\begin{array}{l}40^{\circ} 03^{\prime} \mathrm{N}, 105^{\circ} 36^{\prime} \\
\mathrm{W}\end{array}$ & Subalpine forest-meadow ecotone & 3200 & & & \\
\hline $\begin{array}{l}\text { Southern Wisconsin, } \\
\text { USA }\end{array}$ & $\begin{array}{l}43^{\circ} 02^{\prime} \mathrm{N}, 89^{\circ} 28^{\prime} \mathrm{W} \\
43^{\circ} 21^{\prime} \mathrm{N}, 87^{\circ} 58^{\prime} \mathrm{W}\end{array}$ & $\begin{array}{l}\text { Cold temperate forest and prairie } \\
\text { Alkaline fen and bog }\end{array}$ & $\begin{array}{l}275 \\
245\end{array}$ & 8.0 & 820 & 700 \\
\hline $\begin{array}{l}\text { Coweeta, North Carolina, } \\
\text { USA }\end{array}$ & $\begin{array}{l}35000^{\prime} \mathrm{N}, 83^{\circ} 30^{\prime} \\
\mathrm{W}\end{array}$ & Montane humid temperate forest & $700-850$ & 12.5 & 1829 & 856 \\
\hline $\begin{array}{l}\text { Sevilleta, New Mexico, } \\
\text { USA }\end{array}$ & $\begin{array}{c}34^{\circ} 37^{\prime} \mathrm{N}, 106^{\circ} 54^{\prime} \\
\mathrm{W} \\
34^{\circ} 36^{\prime} \mathrm{N}, 106^{\circ} 69^{\prime} \\
\mathrm{W}\end{array}$ & $\begin{array}{l}\text { Desert grassland and shrubland } \\
\text { Pinyon-juniper woodland }\end{array}$ & $\begin{array}{c}1400-1580 \\
1890\end{array}$ & 13.0 & 222 & 2428 \\
\hline $\begin{array}{l}\text { Hobcaw, South Carolina, } \\
\text { USA }\end{array}$ & $33^{\circ} 20^{\prime} \mathrm{N}, 79^{\circ} 13^{\prime} \mathrm{W}$ & Warm temperate/subtropical forest & $3-4$ & 18.3 & 1295 & 1656 \\
\hline $\begin{array}{l}\text { San Carlos, Amazonas, } \\
\text { Venezuela }\end{array}$ & $1^{\circ} 56^{\prime} \mathrm{N}, 67^{\circ} 03^{\prime} \mathrm{W}$ & Tropical rain forest & 120 & 26.0 & 3560 & 1971 \\
\hline
\end{tabular}

Notes: Annual climate data (mean temperature, mean annual precipitation, and estimated potential evapotranspiration [PET]) are shown for the meteorological station nearest to the main study area at each site. PET was calculated from open-pan evaporation or the equivalent. Four of the sites (Colorado, North Carolina, New Mexico, and South Carolina) were part of the National Science Foundation Long-Term Ecological Research Program.

leaf life span. We have theorized that these relationships are universal at the broadest, continental to global scales (Reich et al. 1992, Reich 1993) because of the functional interdependency among traits, plus the constraints placed by biophysics and natural selection that lead to ecological trade-offs (Coley et al. 1985, Coley 1988, Field and Mooney 1986, Reich et al. 1992, Mulkey et al. 1995).

Thus, we hypothesize that interspecific proportional scaling relationships (e.g., $\log Y=a+b \log X$ ) among leaf traits will be quantitatively similar among diverse biomes, supporting the idea of convergent evolution. Alternatively, leaf trait correlations may occur but vary among vegetation types or along climatic or edaphic gradients. To address these issues we quantified leaf gas exchange rates, SLA, leaf N, and leaf life span, and their relationships for 10-43 species within each of six sites representing different biomes in the Americas. Our sites represent a range of biomes (Table 1) that vary in growing season length, mean air temperature, elevation, water availability, and soil fertility. A brief summary paper (submitted after the original submission of this manuscript) based in part on these same data highlights the idea that the slopes of interspecific trait relationships were similar among biomes and among data sets (Reich et al. 1997). In this paper we take the opportunity to (1) present the biome-specific data, (2) explicitly contrast slopes among sites, (3) compare the elevations of these scaling equations, (4) contrast relationships among functional groups, and (5) make multivariate analyses of species leaf traits; collectively these represent the main objectives of this paper and were beyond the scope of the previous summary publication.

\section{Methods}

Sites were selected to provide a wide range of environmental conditions and terrestrial ecosystem types (Table 1). Two sites (in Colorado and Wisconsin) were located at the ecotone between biomes and include species common to both biomes. At several sites, measurements were made in more than one ecosystem type or study area. The study site in Colorado was located at Niwot Ridge in the Front Range of the Rocky Mountains. Study plots were located in both wet and dry meadow tundra communities at $3510 \mathrm{~m}$ elevation, and in open subalpine forest-alpine meadow transition at $3200 \mathrm{~m}$. The species studied include common conifers, hardwood shrubs, and herbs. Soils at the site were largely coarse-textured Inceptisols. The main study area in Wisconsin was the University of Wisconsin Arboretum, in Madison, Wisconsin (275-m elevation). Study plots were located in a mosaic of natural and restored ecosystems, including forest, hedgerow, savanna and tall grass prairie. Soils were largely mediumtextured silt loams (Alfisols). We also examined species in a cedar-tamarack swamp and adjacent bog at Cedarburg Bog, at the University of Wisconsin-Milwaukee Field Station in Saukville, Wisconsin, $\sim 130 \mathrm{~km}$ northeast of Madison. The climate in southern Wisconsin is humid continental, with cold winters and warm summers. Species studied included a number of common prairie and forest understory forbs, deciduous hardwood and coniferous forest tree species and broadleafed evergreen bog shrubs.

A montane cool temperate forest was studied at the Coweeta Hydrological Laboratory, Otto, North Carolina. We studied naturally growing vegetation in sec- 
ondary forest communities located at $700-850 \mathrm{~m}$ elevation. Precipitation is abundant year-round, and the ratio of precipitation to potential evapotranspiration is high (Table 1). Soils at this site were principally clay loam Ultisols. Species studied included a number of common forest understory forbs, broadleafed deciduous and evergreen hardwood and evergreen coniferous forest tree species.

The lower Carolina coastal plain contains upland pine-dominated forests as well as forested wetlands. We studied selected species from these communities on the Hobcaw Forest in Georgetown County, South Carolina. The climate of the area is maritime, warm temperate/humid subtropical. Annually, potential evapotranspiration exceeds precipitation (Table 1). This difference is greatest during the growing season. Soils are sandy throughout the Hobcaw Forest.

Desert shrubland and pinyon-juniper woodland vegetation were studied (1400-1540 m elevation) in the Sevilleta National Wildlife Refuge, New Mexico. Soils are sandy and classified as Aridosols of HaplargidsTorripsamments construction. The climate is warm and arid. Annually, potential evapotranspiration far exceeds precipitation (Table 1 ).

The tropical rain forest site was located near San Carlos del Rio Negro, Venezuela in the northern Amazon basin. Although much of the data for the Venezuela site have been published before (e.g., Reich et al. 1991), given the design of this study their inclusion in this report is necessary. A total of 24 species were studied in mature stands of three adjacent primary rain forest communities (of Bana, tall Caatinga, and Tierra Firme forest) and in secondary successional stands growing on Tierra Firme sites (Reich et al. 1991, 1994). The different primary forests occur on Spodosols, Ultisols and Oxisols (Reich et al. 1994). The San Carlos region is characterized year-round by abundant rainfall and warm temperatures.

Woody plants and perennial herbaceous species were selected at each site (Table 2) based on the following criteria: we selected species expected to provide a gradient of leaf traits (based on prior general knowledge) and that were relatively abundant at each site. Both broadleaf and needle-leaf species (deciduous or evergreen) were selected at each site when possible.

Since leaf traits vary with leaf age, contrasts of gas exchange rates, $\mathrm{N}$ concentrations and SLA among species were made using leaves of a similar "physiological" age (i.e., ontogenetic stage). We used fully expanded young to medium-aged leaves of all species, which corresponds to the period when many leaf traits are relatively stable (Reich et al. 1991). Gas exchange measurements were made during the growing season between 1987 and 1993. To minimize the potentially confounding influence of shade, we tried to select "sun" leaves growing in relatively open conditions for all species at all sites. Measurements were made on open-grown plants in all herbaceous dominated com- munities and usually were made for open-grown trees or shrubs, or for mature trees in the upper canopy. Although leaf light microenvironment has a large impact on leaf traits, especially SLA (e.g., Ellsworth and Reich 1992), the interspecific differences in leaf traits in this study were large enough (often $25-50 \times$ differences) that smaller intraspecific differences due to variation in leaf microenvironment (usually by factors of less than two) would not have been significant.

Measurements of photosynthetic $\mathrm{CO}_{2}$ assimilation and leaf water vapor conductance were made under ambient conditions at all sites with a portable leaf chamber and infrared gas analyzer operated in the differential mode (ADC model LCA-2, Hoddesdon, England). Measurements were made at mid- to late morning (0800-1100 local time) when the following conditions were met: near full sunlight, relatively nonlimiting vapor pressure deficits or temperatures. Thus, sampling was designed so that measurements were taken to closely reflect leaf photosynthetic capacity in the field at ambient $\mathrm{CO}_{2}$ concentration (Reich et al. 1991, Ellsworth and Reich 1992). We took at least 10 (but usually more) measurements per species from several individuals at each site, then averaged these for subsequent analyses.

After measuring gas exchange rates, foliage was harvested. The projected surface area of either the leaf tissue or its silhouette was assessed by a digital image analysis system (Decagon Instruments, Pullman, Washington). Total surface area was also calculated based on the shape of each leaf type. The results were similar if total rather than projected surface area was used, although the quantitative relations differ. Given that projected area was measured, while total surface area was estimated indirectly, data are expressed on a projected area basis. Since SLA is by definition related to leaf thickness and density (Abrams et al. 1994, Garnier and Laurent 1994) we will use the terms leaf thickness, density, and SLA to convey roughly the same information.

In order to determine leaf life spans of broad-leaved species, leaf birth and death were monitored (using tagging and/or drawings) for numerous leaves of at least several plants per species (see Reich et al. 1991). In some instances, observations of leaf phenology were used instead of direct measurement. For coniferous species with long-lived foliage, the average needle longevity was calculated by counting the number of annual cohorts with at least $50 \%$ of their needles retained on the branch.

In statistical analyses, individual data points represent average values for a single species at a single site. A linear regression model was generally inappropriate to describe the relations between pairs of leaf traits, because the data were not normally distributed (Shapiro-Wilk W test, all leaf traits $P<0.0001$ ), the relationships were often not linear, and there was patterned heteroscedasticity in the residuals with the ab- 
TABLE 2. List of species, sites, and physiological data (mean per species per site).

\begin{tabular}{|c|c|c|c|c|c|c|c|c|c|}
\hline Location & Species & $\begin{array}{l}\text { Func- } \\
\text { tional } \\
\text { group }\end{array}$ & $\begin{array}{l}\text { Leaf } \\
\text { type }\end{array}$ & $\begin{array}{l}\text { Leaf } \\
\text { life } \\
\text { span } \\
(\mathrm{mo})\end{array}$ & $\begin{array}{l}\text { SLA } \\
\left(\mathrm{cm}^{2} / \mathrm{g}\right)\end{array}$ & $\begin{array}{l}\text { Leaf } N \\
(\mathrm{mg} / \mathrm{g})\end{array}$ & $\begin{array}{c}A_{\text {mass }} \\
(\mathrm{nmol} \cdot \\
\left.\mathrm{g}^{-1} \cdot \mathrm{s}^{-1}\right)\end{array}$ & $\begin{array}{c}A_{\text {area }} \\
(\mu \mathrm{mol} \cdot \\
\left.\mathrm{m}^{-2} \cdot \mathrm{s}^{-1}\right)\end{array}$ & $\begin{array}{c}G_{s} \\
(\mathrm{mmol} \cdot \\
\left.\mathrm{m}^{-2} \cdot \mathrm{s}^{-1}\right)\end{array}$ \\
\hline \multirow[t]{10}{*}{ Colorado } & Acomastylis rosii & forb & broad & 2.0 & 119.0 & 25.0 & 120.4 & 10.8 & 673 \\
\hline & Bistorta bistortoides & forb & broad & 2.0 & 121.0 & 39.0 & 136.0 & 11.1 & 512 \\
\hline & Psychrophila leptosepala & forb & broad & 2.5 & 126.0 & 29.0 & 110.0 & 8.7 & 462 \\
\hline & Salix glauca & shrub & broad & 3.0 & 122.8 & 26.3 & 124.3 & 10.2 & 481 \\
\hline & Salix planifolia & shrub & broad & 3.0 & 123.0 & 26.7 & 151.4 & 12.2 & 542 \\
\hline & Vaccinium myrtillus & shrub & broad & 3.5 & 171.0 & 23.4 & 104.5 & 6.1 & 249 \\
\hline & Arctostaphylos uva-ursi & shrub & broad & 18.0 & 78.4 & 12.3 & 31.8 & 4.4 & 191 \\
\hline & Pinus flexilis & tree & needle & 36.0 & 27.3 & 11.2 & 25.9 & 9.7 & 257 \\
\hline & Picea engelmanii & tree & needle & 90.0 & 32.7 & 10.3 & 11.6 & 3.5 & 96 \\
\hline & Abies lasiocarpa & tree & needle & 96.0 & 39.3 & 10.3 & 11.5 & 2.9 & 81 \\
\hline \multirow[t]{14}{*}{ North Carolina } & Podophyllum peltatum & forb & broad & 3.0 & 323.0 & 41.5 & 261.0 & 6.5 & 169 \\
\hline & Veratrum parviflorum & forb & broad & 3.6 & 297.0 & $\ldots$ & 145.0 & 4.4 & 131 \\
\hline & Helianthus microcephalus & forb & broad & 4.1 & 251.0 & 26.2 & 259.6 & 10.5 & 536 \\
\hline & Robinia pseudoacacia & tree & broad & 4.6 & 264.0 & 43.3 & 243.1 & 9.4 & 297 \\
\hline & Eupatorium rugesum & forb & broad & 4.8 & 469.0 & 39.2 & 382.0 & 6.8 & 224 \\
\hline & Acer rubrum & tree & broad & 5.2 & 160.0 & 18.4 & 125.0 & 7.7 & 237 \\
\hline & Liriodendron tulipifera & tree & broad & 5.3 & 165.0 & 22.4 & 170.0 & 10.2 & 450 \\
\hline & Quercus coccinea & tree & broad & 5.7 & 118.0 & 17.3 & 111.0 & 9.7 & 338 \\
\hline & Galax aphylla & forb & broad & 18.0 & 125.0 & 8.9 & 78.0 & 4.9 & 87 \\
\hline & Pinus strobus & tree & needle & 21.0 & 92.0 & 13.5 & 50.6 & 6.0 & 198 \\
\hline & Pinus rigida & tree & needle & 33.0 & 48.9 & 11.6 & 55.7 & 11.3 & 317 \\
\hline & Kalmia latifolia & shrub & broad & 36.0 & 94.5 & 11.5 & 43.3 & 4.8 & 172 \\
\hline & Rhododendron maximum & tree & broad & 48.0 & 48.9 & 8.6 & 33.9 & 6.8 & 136 \\
\hline & Tsuga canadensis & tree & needle & 60.0 & 81.6 & 9.9 & 43.9 & 5.5 & 141 \\
\hline \multirow[t]{11}{*}{ New Mexico } & Baccharis angustifolia & shrub & broad & 2.5 & 105.0 & 19.5 & 197.8 & 18.7 & 575 \\
\hline & Eleagnus angustifolia & tree & broad & 3.0 & 124.0 & 39.8 & 175.1 & 14.1 & 404 \\
\hline & Populus fremontii & tree & broad & 3.0 & 83.8 & 16.6 & 120.0 & 14.4 & 495 \\
\hline & Gutierrezia sarothrae & forb & broad & 6.0 & 33.9 & 16.8 & 59.0 & 14.7 & 641 \\
\hline & Atriplex canescens & shrub & broad & 7.0 & 39.7 & 21.0 & 79.0 & 17.1 & 350 \\
\hline & Prosopis glandulosa & shrub & broad & 7.0 & 51.2 & 24.9 & 92.0 & 17.9 & 367 \\
\hline & Quercus turbinella & shrub & broad & 8.0 & 66.0 & 15.4 & & & \\
\hline & Larrea tridentata & shrub & broad & 12.0 & 39.6 & 19.4 & 85.0 & 18.3 & 330 \\
\hline & Pinus edulis & tree & needle & 66.0 & 24.5 & 10.6 & 12.7 & 4.1 & 103 \\
\hline & Juniperus monosperma & tree & needle & 78.0 & 12.8 & 11.7 & 11.6 & 7.7 & 151 \\
\hline & Juniperus monosperma & tree & needle & 98.0 & 12.1 & $\cdots$ & & $\cdots$ & 96 \\
\hline \multirow[t]{10}{*}{ South Carolina } & Pterocaulon pycnostachyum & forb & broad & 5.1 & 123.6 & 19.7 & 101.3 & 7.8 & 365 \\
\hline & Taxodium distichum & tree & needle & 6.5 & 78.0 & 11.9 & 64.8 & 8.3 & 281 \\
\hline & Vaccinium corymbosum & shrub & broad & 7.5 & 97.9 & 12.3 & 60.6 & 6.2 & 145 \\
\hline & Quercus laevis & tree & broad & 7.6 & 97.6 & 11.5 & 65.8 & 6.8 & 181 \\
\hline & $\begin{array}{l}\text { Quercus virginiana var. } \\
\text { geminata }\end{array}$ & tree & broad & 11.0 & 62.9 & 15.4 & 80.4 & 12.8 & 361 \\
\hline & Vaccinium arboreum & shrub & broad & 11.0 & 80.1 & 12.1 & 52.5 & 6.4 & 166 \\
\hline & Persea borbonia & tree & broad & 18.0 & 90.0 & 16.4 & 62.0 & 6.7 & 203 \\
\hline & Lyonia lucida & shrub & broad & 19.5 & 42.1 & 9.2 & 27.2 & 6.2 & 185 \\
\hline & Pinus serotina & tree & needle & 27.0 & 35.9 & 8.2 & 15.8 & 4.1 & 94 \\
\hline & Pinus palustris & tree & needle & 32.0 & 39.2 & 8.2 & 16.7 & 3.9 & 67 \\
\hline \multirow[t]{18}{*}{ Venezuela } & Manihot esculenta & shrub & broad & 1.5 & 275.1 & 39.8 & 467.9 & 16.9 & 1132 \\
\hline & Solanum straminifolia & shrub & broad & 2.0 & 287.4 & 39.2 & 378.5 & 13.5 & 1556 \\
\hline & Cecropia ficifolia & tree & broad & 2.7 & 144.6 & 24.7 & 252.2 & 17.7 & 2272 \\
\hline & Vismia lauriformis & tree & broad & 4.5 & 114.3 & 17.9 & 159.3 & 13.8 & 889 \\
\hline & Clidemia sericea & tree & broad & 5.1 & 126.4 & 16.5 & 115.5 & 9.1 & 597 \\
\hline & Vismia japurensis & tree & broad & 6.9 & 105.4 & 16.4 & 140.4 & 12.8 & 975 \\
\hline & Bellucia grossularioides & tree & broad & 8.4 & 78.1 & 16.9 & 111.5 & 14.0 & 1707 \\
\hline & Goupia glabra & tree & broad & 11.4 & 129.9 & 15.1 & 99.0 & 7.8 & 300 \\
\hline & Neea obovata & tree & broad & 12.0 & 103.1 & 18.4 & 65.0 & 6.4 & 479 \\
\hline & Miconia dispar & tree & broad & 12.0 & 90.3 & 15.9 & 91.8 & 10.3 & 1009 \\
\hline & Retiniphyllum truncatum & tree & broad & 16.5 & 82.8 & 6.8 & 46.5 & 5.6 & 490 \\
\hline & Rhodognaphalopsis humilis & tree & broad & 24.0 & 75.2 & 7.8 & 47.2 & 6.2 & 693 \\
\hline & Protium sp. & tree & broad & 33.0 & 86.6 & 8.6 & 34.7 & 4.0 & 321 \\
\hline & Aspidosperma album & tree & broad & 33.0 & 82.6 & 10.7 & 52.2 & 6.5 & 411 \\
\hline & Protium sp. & tree & broad & 35.0 & 82.0 & 17.7 & 67.2 & 8.2 & 381 \\
\hline & Caraipa heterocarpa & tree & broad & 38.5 & 67.8 & 9.3 & 38.8 & 5.7 & 241 \\
\hline & Ocotea costulata & tree & broad & 39.0 & 76.8 & 15.0 & 44.9 & 5.9 & 329 \\
\hline & Licania heteromorpha & tree & broad & 40.0 & 67.3 & 13.0 & 53.8 & 8.0 & 378 \\
\hline
\end{tabular}


TABle 2. Continued.

\begin{tabular}{|c|c|c|c|c|c|c|c|c|c|}
\hline Location & Species & $\begin{array}{l}\text { Func- } \\
\text { tional } \\
\text { group }\end{array}$ & $\begin{array}{l}\text { Leaf } \\
\text { type }\end{array}$ & $\begin{array}{l}\begin{array}{c}\text { Leaf } \\
\text { life } \\
\text { span } \\
(\mathrm{mo})\end{array}\end{array}$ & $\begin{array}{c}\text { SLA } \\
\left(\mathrm{cm}^{2} / \mathrm{g}\right)\end{array}$ & $\begin{array}{c}\text { Leaf } N \\
(\mathrm{mg} / \mathrm{g})\end{array}$ & $\begin{array}{c}A_{\text {mass }} \\
(\mathrm{nmol} \cdot \\
\left.\mathrm{g}^{-1} \cdot \mathrm{s}^{-1}\right)\end{array}$ & $\begin{array}{c}A_{\text {area }} \\
(\mu \mathrm{mol} \cdot \\
\left.\mathrm{m}^{-2} \cdot \mathrm{s}^{-1}\right)\end{array}$ & $\begin{array}{c}G_{s} \\
\left(\mathrm{mmol}^{-2} \cdot \mathrm{s}^{-1}\right) \\
\mathrm{m}^{-1}\end{array}$ \\
\hline & Eperua purpurea & tree & broad & 43.0 & ... & 15.2 & 55.1 & $\ldots$ & 209 \\
\hline & Eperua leucantha & tree & broad & 44.0 & 95.1 & 12.5 & 35.1 & 3.7 & 173 \\
\hline & Leguminosae sp. & tree & broad & 50.0 & 72.1 & 21.4 & 47.7 & 6.7 & 235 \\
\hline & Micrandra sprucei & tree & broad & 50.0 & 58.4 & 10.8 & 43.3 & 7.4 & 298 \\
\hline & Micropholis maguirei & tree & broad & 50.5 & 55.3 & 8.0 & $\ldots$ & . & \\
\hline & Protium sp. & tree & broad & 51.0 & 58.1 & 10.3 & 33.0 & 5.7 & 274 \\
\hline \multirow[t]{43}{*}{ Wisconsin } & Caulophyllum thalictroides & forb & broad & $\ldots$ & 425.0 & 58.2 & 254.0 & 5.9 & 134 \\
\hline & Arisaema triphyllum & forb & broad & $\ldots$ & 380.0 & 63.6 & & & 90 \\
\hline & Dentaria laciniata & forb & broad & 1.1 & 297.0 & 53.0 & 432.0 & 14.2 & 227 \\
\hline & Erythronium americanum & forb & broad & 1.9 & 222.0 & 42.0 & 263.0 & 11.9 & 359 \\
\hline & Silphium terebinthinaceum & forb & broad & 3.0 & 133.0 & 14.4 & 175.0 & 13.4 & 615 \\
\hline & Podophyllum peltatum & forb & broad & 3.0 & 309.0 & 44.7 & 244.0 & 7.9 & 164 \\
\hline & Baptisia leucophaea & forb & broad & 3.5 & 106.3 & 35.9 & 159.0 & 15.0 & 481 \\
\hline & Trillium grandiflora & forb & broad & 3.7 & 357.0 & 51.6 & 209.0 & 5.8 & 499 \\
\hline & Echinacea purpurea & forb & broad & 4.0 & 128.5 & 15.0 & 122.9 & 9.8 & 480 \\
\hline & Silphium integrifolium & forb & broad & 4.0 & 116.3 & 16.6 & 116.0 & 10.0 & 478 \\
\hline & Sanguinaria canadensis & forb & broad & 4.0 & 321.0 & 53.6 & 255.0 & 7.9 & 208 \\
\hline & Populus deltoides & tree & broad & 5.0 & 110.0 & 23.6 & 162.0 & 14.8 & 595 \\
\hline & Populus tremuloides & tree & broad & 5.0 & 121.0 & 22.1 & 142.0 & 11.9 & 431 \\
\hline & Juglans nigra & tree & broad & 5.0 & 305.0 & 29.6 & 175.0 & 5.8 & 162 \\
\hline & Betula nigra & tree & broad & 5.2 & 118.0 & 22.8 & 118.0 & 10.0 & 296 \\
\hline & Acer rubrum & tree & broad & 5.5 & 166.0 & 21.0 & 108.0 & 6.5 & $\ldots$ \\
\hline & Acer saccharum & tree & broad & 5.5 & 125.0 & 18.5 & 100.0 & 7.6 & $\ldots$ \\
\hline & Prunus serotina & tree & broad & 5.5 & 99.0 & 20.7 & 125.0 & 11.7 & $\ldots$ \\
\hline & Celtis occidentales & tree & broad & 5.5 & 121.0 & 23.9 & 117.0 & 9.7 & 403 \\
\hline & Carya ovata & tree & broad & 5.6 & 112.0 & 19.8 & 91.0 & 8.4 & 316 \\
\hline & Cornus florida & tree & broad & 5.6 & 108.0 & 14.0 & 85.0 & 7.3 & \\
\hline & Fraxinus americana & tree & broad & 5.7 & 138.0 & 19.8 & 121.0 & 8.6 & 408 \\
\hline & Ulmus americana & tree & broad & 5.8 & 84.0 & 19.3 & 116.0 & 13.8 & 454 \\
\hline & Betula pumila & tree & broad & 6.0 & 93.0 & 15.1 & 67.2 & 7.6 & 255 \\
\hline & Ilex verticillata & shrub & broad & 6.0 & 110.4 & 15.5 & 72.8 & 6.6 & 215 \\
\hline & Larix decidua & tree & needle & 6.0 & 139.0 & 21.0 & 102.0 & 8.9 & 250 \\
\hline & Quercus ellipsoidalis & tree & broad & 6.0 & 95.0 & 21.0 & 125.0 & 13.2 & \\
\hline & Quercus macrocarpa & tree & broad & 6.0 & 114.0 & 23.5 & 149.4 & 13.6 & 598 \\
\hline & Larix laricina & tree & needle & 6.0 & 83.3 & 14.3 & 52.3 & 6.7 & 445 \\
\hline & Cornus racemosa & shrub & broad & 6.0 & 123.0 & 14.5 & 95.0 & 7.0 & 250 \\
\hline & Lonicera $\times$ bella & shrub & broad & 6.7 & 120.0 & 19.0 & 118.0 & 9.8 & 320 \\
\hline & Rhamnus cathartica & shrub & broad & 6.8 & 115.0 & 23.0 & 135.0 & 11.0 & 440 \\
\hline & Andromeda glaucophylla & shrub & broad & 13.0 & 75.8 & 13.9 & 70.3 & 9.3 & 309 \\
\hline & Chamaedaphne calyculata & shrub & broad & 13.0 & 114.9 & 11.9 & 70.1 & 6.1 & 299 \\
\hline & Pinus strobus & tree & needle & 21.0 & 74.0 & 17.0 & 45.0 & 6.1 & 205 \\
\hline & Pinus banksiana & tree & needle & 27.0 & 41.0 & 12.4 & 31.0 & 7.6 & 231 \\
\hline & Juniper virginiana & tree & needle & 27.0 & 30.0 & 16.4 & 33.0 & 6.5 & $\ldots$ \\
\hline & Pinus sylvestris & tree & needle & 27.0 & 34.0 & 13.9 & 35.0 & 10.3 & $\ldots$ \\
\hline & Sarracenia purpurea & forb & broad & 30.0 & 78.1 & 11.4 & 22.8 & 2.9 & 144 \\
\hline & Pinus resinosa & tree & needle & 36.0 & 34.0 & 11.7 & 24.0 & 6.3 & $\ldots$ \\
\hline & Thuja occidentalis & tree & needle & 48.0 & 44.8 & 7.6 & 32.0 & 7.2 & 163 \\
\hline & Picea glauca & tree & needle & 60.0 & 35.0 & 12.4 & 23.0 & 6.6 & 190 \\
\hline & Picea mariana & tree & needle & 60.0 & 34.0 & 12.1 & 37.0 & 9.2 & $\ldots$ \\
\hline
\end{tabular}

Notes: Data shown are specific leaf area (SLA), mass-based leaf nitrogen (leaf $N_{\text {mass }}$ ), mass-based net photosynthetic capacity $\left(A_{\text {mass }}\right)$, area-based net photosynthetic capacity $\left(A_{\text {area }}\right)$, and leaf diffusive conductance $\left(G_{s}\right)$ at photosynthetic capacity.

solute value of the residuals increasing as a function of the character value. Therefore, we used logarithmic (base-10) transformations of the data, which generally normalized the data distribution, linearized the regression functions, and stabilized the error term variances.

When any given leaf trait was used in regression as the independent variable, there was no random sampling variation associated with it because the species were selected based on a priori knowledge to provide a roughly continuous range of leaf traits. Based on these considerations, Type I (sometimes also called Model I) regression is appropriate (Steel and Torrie 1980, Sokal and Rohlf 1995). Given that there is both biological and error variation associated with our measures of all traits used as independent variables, and that not all relations involve direct causality, we also analyzed these relationships using a Type II regression approach involving bivariate analyses (Steel and Torrie 1980, 
TABLE 3. Summary of multiple regression analyses for leaf traits in relation to site and other leaf traits.

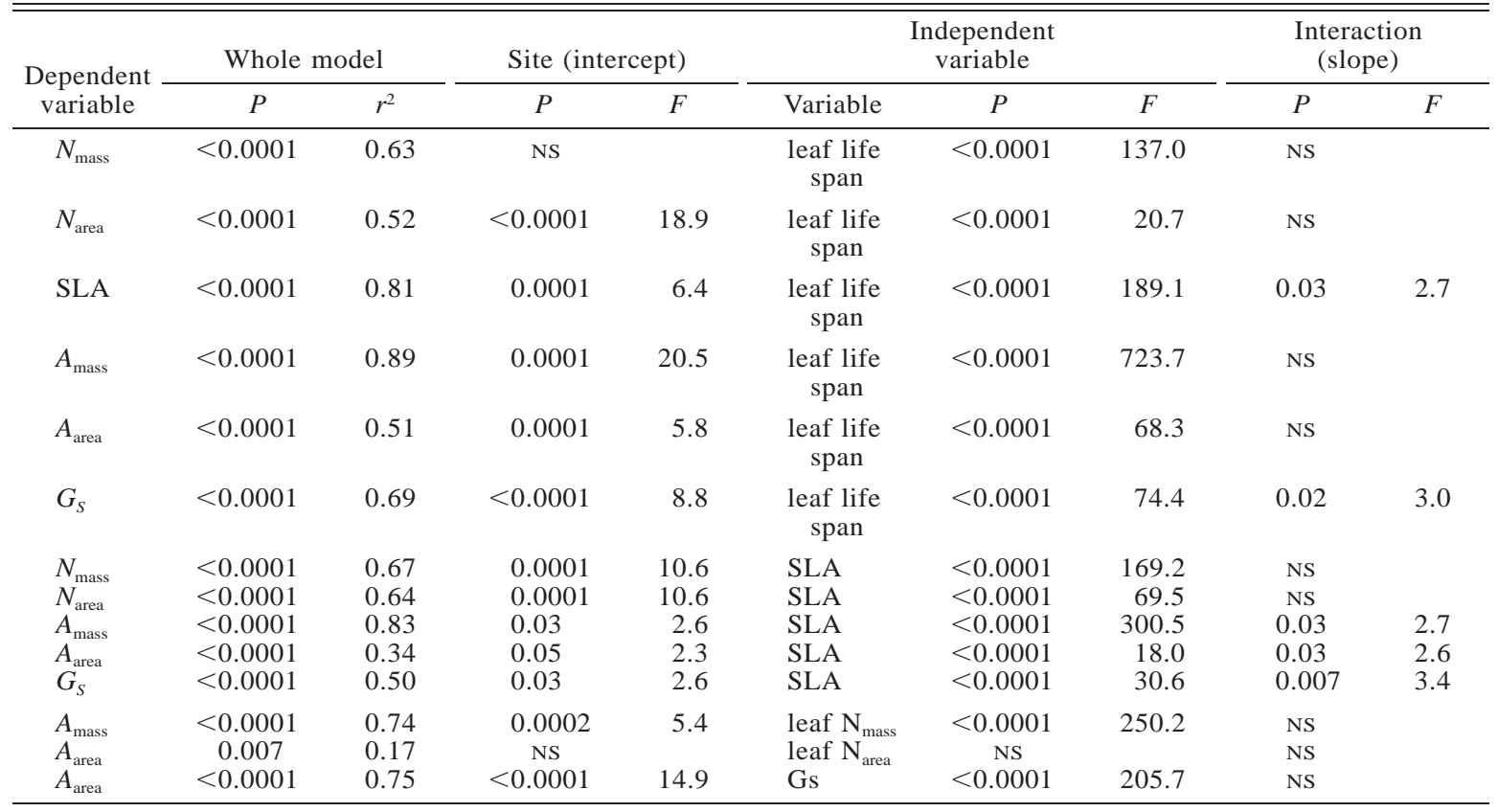

Notes: The interaction term serves as a test for slope differences among sites. If the interaction term was not significant it was dropped from the model, and in those cases the site term serves as a test for differences among sites in the elevation of the line.

Sokal and Rohlf 1995). The significance levels and the fits of these Type II regression analyses were almost identical to those obtained using Type I regression. Moreover, the bivariate normal density ellipses $(P=$ 0.95 ) were very similar to the $95 \%$ confidence intervals for individual observations (predictions of individuals). Thus, despite some uncertainty about whether the Type II or Type I model is more appropriate, the results are similar if analyzed either way. Data were further analyzed using ANCOVA, separate slopes analyses, multiple regression, and principal components analyses (PCA) (JMP Statistical Software, SAS Institute). Analyses were made (using linear contrasts of the transformed variables) to test whether the slopes of the lines varied among sites (i.e., the interaction between site and independent traits, Table 3 ). If they did not differ significantly, the interaction term was removed from the model and so-called "same slopes" analyses were used to test for intersite differences among regression lines (interpreted in this paper as a difference in the elevation of the total line). If the slopes did differ significantly ANCOVA was used to test for differences among sites in the dependent variable (Table 4) at the grand mean of the covariate (the independent variable). PCA was also conducted to simultaneously explore species variation in multiple leaf traits.

\section{RESUlts}

Leaf $N_{\text {mass }}, S L A$, and gas exchange vs. leaf life span

Leaf $N_{\text {mass }}$, SLA, and life span varied nearly $10 \times$, $40 \times$, and $90 \times$, respectively, among species (Fig. 1,
Table 2). At every site (Fig. 1) leaf $N_{\text {mass }}$ declined significantly (for all results reported, $P<0.001$ unless stated otherwise) in relation to increasing leaf life span (mean $r^{2}=0.60$ ) and this relationship was similar for all data pooled. Sites did not differ significantly in the slope or elevation of the $N_{\text {mass }}$ : leaf life span relationship (Table 3$)$. Leaf $N_{\text {area }}$ increased significantly $(P<$ $0.05)$ with increasing leaf life span at three of the six sites (Fig. 1), and was very weakly related to leaf life span using all data pooled.

At every site SLA declined significantly with increasing leaf life span (Fig. 1) and the relationship varied among sites (Fig. 1, Table 3). At any given leaf life span, SLA was highest in humid temperate and tropical forests (North Carolina and Venezuela) and lowest in desert shrubland (New Mexico) (Fig. 1, Table 4).

$A_{\text {mass }}$ varied 40 -fold (from 12 to $468 \mathrm{nmol} \cdot \mathrm{g}^{-1} \cdot \mathrm{s}^{-1}$ ) among all species (Fig. 1, Table 2). $A_{\text {mass }}$ decreased markedly with increasing leaf life span at each of the six sites (average $r^{2}=0.88$ ) and for all data pooled $\left(r^{2}\right.$ $=0.78$ ). The slopes of these relationships did not differ among sites, but the elevations of the lines did (Table 3). Overall, species from humid temperate (North Carolina) and tropical forests (Venezuela) had higher $A_{\text {mass }}$ on average at any given leaf life span than those from subtropical (South Carolina), desert shrubland (New Mexico) or alpine/subalpine sites (Colorado) (Fig. 1, Tables 3 and 4).

Area-based net photosynthetic rate $\left(A_{\text {area }}\right)$ varied 
TABLE 4. Site means of physiological measures of leaf traits.

\begin{tabular}{lccccccc}
\hline \hline & & \multicolumn{9}{c}{ Site } \\
\cline { 3 - 7 } \multicolumn{1}{c}{ Leaf trait } & Value & Colorado & Wisconsin & $\begin{array}{c}\text { North } \\
\text { Carolina }\end{array}$ & $\begin{array}{c}\text { New } \\
\text { Mexico }\end{array}$ & $\begin{array}{c}\text { South } \\
\text { Carolina }\end{array}$ & Venezuela \\
\hline Specific leaf area $\left(\mathrm{cm}^{2} / \mathrm{g}\right)$ & absolute & 81.3 & 107.2 & 144.5 & 41.7 & 69.2 & 95.5 \\
& adjusted & 74.1 & 93.3 & 147.9 & 44.7 & 75.9 & 117.4 \\
Leaf $N_{\text {mass }}(\mathrm{mg} / \mathrm{g})$ & absolute & 19.1 & 19.5 & 17.8 & 18.2 & 12.0 & 14.5 \\
& adjusted & 18.2 & 17.8 & 18.6 & 17.8 & 12.9 & 17.0 \\
Leaf $N_{\text {area }}\left(\mathrm{g} / \mathrm{m}^{2}\right)$ & absolute & 2.41 & 1.80 & 1.29 & 3.83 & 1.74 & 1.53 \\
& adjusted & 2.36 & 1.87 & 1.26 & 3.88 & 1.69 & 1.44 \\
$A_{\text {mass }}\left(\mathrm{nmol} \cdot \mathrm{g}^{-1} \cdot \mathrm{s}^{-1}\right)$ & absolute & 57.4 & 95.5 & 107.2 & 66.1 & 46.8 & 77.6 \\
& adjusted & 51.3 & 77.6 & 112.2 & 61.7 & 53.7 & 107.2 \\
$A_{\text {area }}\left(\mu \mathrm{mol} \cdot \mathrm{m}^{-2} \cdot \mathrm{s}^{-1}\right)$ & absolute & 7.1 & 8.7 & 7.1 & 12.9 & 6.6 & 8.1 \\
Leaf life span $(\mathrm{mo})$ & adjusted & 6.9 & 8.2 & 7.3 & 12.7 & 6.9 & 9.0 \\
\hline
\end{tabular}

Notes: Absolute values were back-transformed from means of transformed values. Adjusted values were back-transformed from least-squares means adjusted for leaf life span using analysis of covariance.

6-fold among species (from 3 to $19 \mu \mathrm{mol} \cdot \mathrm{m}^{-2} \cdot \mathrm{s}^{-1}$ ). There was a significant negative relationship between $A_{\text {area }}$ and leaf life span in five of six sites (Fig. 1) and for all data pooled $\left(r^{2}=0.36\right)$. The slopes did not differ significantly but the elevations of the lines did (Table $3)$. At any given leaf life span species from desert shrubland (New Mexico) tended to have higher $A_{\text {area }}$ than those from any other biome. Their lower SLA more than compensates for slightly lower $A_{\text {mass }}$ in terms of effects on $A_{\text {area }}$ (given that $A_{\text {area }}=A_{\text {mass }} /$ SLA).

The relationship between leaf diffusive conductance $\left(G_{s}\right)$ and leaf life span was not significant in humid temperate forest (North Carolina), but was significant at all other sites and for pooled data (Fig. 2). Tropical rain forest species tended to have the highest $G_{s}$ for any given leaf life span (Fig. 2). At all sites and for pooled data, $A_{\text {area }}$ increased with $G_{s}$ (average $r^{2}=0.75$, Fig. 2). The slopes did not differ among sites, but the elevations of the lines did (Table 3 ). At any given $G_{s}$, $A_{\text {area }}$ tended to be highest in desert shrubland (the most arid study area) and lowest in tropical rain forest (a humid area).

\section{Leaf $N$ and gas exchange rates vs. SLA}

Leaf $N_{\text {mass }}$ was positively correlated to SLA at each of the six sites (mean $r^{2}=0.66$ ) and for all data pooled $\left(r^{2}=0.50\right.$, Fig. 3). The slopes did not differ among sites, but the elevations of the lines did (Table 3). For any given SLA, $N_{\text {mass }}$ tended to be highest in the desert shrubland site. Leaf $A_{\text {mass }}$ increased with SLA for all data pooled $\left(r^{2}=0.74\right)$ and at each site ( $r^{2}$ ranged from 0.78 to 0.86 ) (Fig. 3). The slopes were different among sites $(P=0.03)$, but this interaction explained a small fraction of the total variation in $A_{\text {mass }}$, compared to SLA (Table 3). At any given SLA, species in desert shrubland (New Mexico) had the highest $A_{\text {mass }}$, with species from other sites having lower values (Fig. 3, Table 4).

Leaf $N_{\text {area }}$ decreased with increasing SLA in five of six sites and for all data pooled $\left(r^{2}=0.45\right.$, Fig. 3$)$. At any given SLA, species from the desert shrubland (New Mexico) site had higher $N_{\text {area }}$ than other species (Table 3 , Fig. 3). $A_{\text {area }}$ was not significantly correlated with SLA at 5 of 6 sites or for all data pooled (data not shown).

\section{Leaf gas exchange rates and leaf $N$}

At all sites and for pooled data, there were highly significant relationships between $A_{\text {mass }}$ and $N_{\text {mass }}$ (Fig. $4, r^{2}$ ranged from 0.7 to 0.9 ). The $A_{\text {mass }}-N_{\text {mass }}$ slopes were not different among sites, but the elevations of the lines were significantly different. At any given leaf $N_{\text {mass }}, A_{\text {mass }}$ tended to be higher in humid temperate and tropical rain forest and lower in desert shrub and alpine tundra/subalpine forest. The regression between $A_{\text {area }}$ and $N_{\text {area }}$ (Fig. 4) was not significant in five sites, was weakly significant $\left(r^{2}=0.26, P=0.09\right)$ in North Carolina, and was significant $(P<0.01)$ but with a low $r^{2}(0.07)$ for all data pooled.

\section{Leaf traits and climate}

Multiple regression was used to evaluate whether leaf traits were significantly related to combinations of climate variables and other leaf traits. Although there were significant models that included either mean annual precipitation or mean annual temperature, the climate measure that was generally best related to leaf traits was an index of site moisture balance (precipitation minus potential evapotranspiration). SLA, $A_{\text {mass }}$, and $G_{s}$ were all significantly related to the additive combination of site moisture balance index (positively) and leaf life span (negatively) (Table 5, Fig. 5). Thus, SLA, $A_{\text {mass }}$, and $G_{s}$ tend to be greater in moister rather than drier environments once leaf life span was considered. In contrast, $N_{\text {area }}$ was negatively related to site moisture balance and positively related to leaf life span (Table 5). 

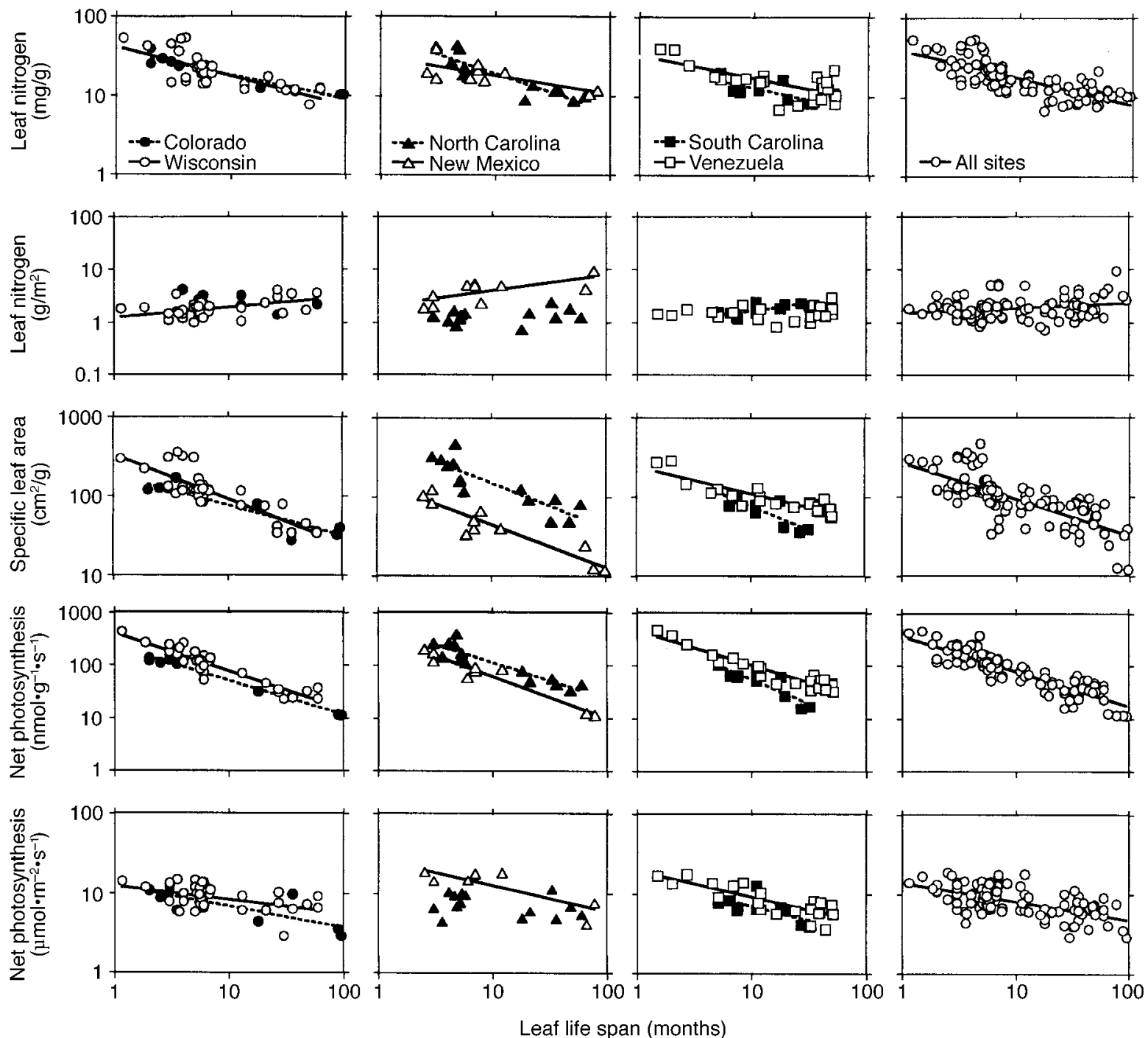

FIG. 1. Mass-based leaf $\mathrm{N}$ concentration $\left(N_{\text {mass }}\right)$, area-based leaf $\mathrm{N}$ content $\left(N_{\text {area }}\right)$, specific leaf area (SLA), mass-based net photosynthetic rate $\left(A_{\text {mass }}, \mathrm{nmol} \cdot \mathrm{g}^{-1} \cdot \mathrm{s}^{-1}\right)$, and area-based net photosynthetic rate $\left(A_{\text {area }}, \mu \mathrm{mol} \cdot \mathrm{m}^{-2} \cdot \mathrm{s}^{-1}\right)$ in relation to leaf life span for plant species in six diverse ecosystems. Ecosystems are arranged from left to right panels by ranking (low to high) their mean annual temperature (alpine tundra/subalpine forest, Colorado [CO]; cold temperate forest, bog, and prairie, Wisconsin [WI]; mesic temperate forest, North Carolina [NC]; desert and scrub, New Mexico [NM]; warm temperate forest, South Carolina [SC]; tropical rain forest, Venezuela [VEN]). All regressions for Figs. 1-4 were significant at $P<0.001$ (based on linear regressions of base-10 logarithmically transformed data) unless shown otherwise immediately following the correlation coefficient. Correlation coefficients $\left(r^{2}\right)$ for leaf $N_{\text {mass }}$ vs. leaf life span: CO $\left(r^{2}=0.92\right)$, WI $\left(r^{2}=0.54\right)$, NC $\left(r^{2}\right.$ $=0.73)$, NM $\left(r^{2}=0.51\right), \mathrm{SC}\left(r^{2}=0.46\right)$, and VEN $\left(r^{2}=0.51\right)$; for leaf $N_{\text {area }}$ vs. leaf life span: CO $\left(r^{2}=0.15, P=0.27\right)$, WI $\left(r^{2}=0.25\right)$, NC $\left(r^{2}=0.07, P=0.38\right)$, NM $\left(r^{2}=0.49\right)$, SC $\left(r^{2}=0.48\right)$, and VEN $\left(r^{2}=0.02, P=0.52\right)$; for SLA vs. leaf life span: $\mathrm{CO}\left(r^{2}=0.84\right)$, WI $\left(r^{2}=0.72\right)$, NC $\left(r^{2}=0.76\right), \mathrm{NM}\left(r^{2}=0.81\right)$, SC $\left(r^{2}=0.74\right)$, and VEN $\left(r^{2}=0.79\right)$; for leaf $A_{\text {mass }}$ vs. leaf life span: CO $\left(r^{2}=0.98\right)$, WI $\left(r^{2}=0.85\right)$, NC $\left(r^{2}=0.84\right)$, NM $\left(r^{2}=0.94\right)$, SC $\left(r^{2}=0.77\right)$, and VEN $\left(r^{2}\right.$ $=0.90)$; and for $A_{\text {area }}$ vs. leaf life span: CO $\left(r^{2}=0.64\right)$, WI $\left(r^{2}=0.21\right)$, NC $\left(r^{2}=0.07, P=0.34\right)$, NM $\left(r^{2}=0.65\right)$, SC $\left(r^{2}=0.41\right)$, and VEN $\left(r^{2}=0.69\right)$. Regression relationships for pooled data: $\log \left(N_{\text {mass }}\right)=1.57-0.34 \log (\operatorname{leaf} \operatorname{life}$ span $), r^{2}$ $=0.59 ; \log \left(N_{\text {area }}\right)=0.17+0.09 \log ($ leaf life span $), r^{2}=0.05, P=0.02 ; \log ($ SLA $)=2.43-0.46 \log (\operatorname{leaf} \operatorname{life}$ span $), r^{2}$ $=0.57 ; \log \left(A_{\text {mass }}\right)=2.56-0.66 \log ($ leaf life span $), r^{2}=0.78 ; \log \left(A_{\text {area }}\right)=1.15-0.23 \log ($ leaf life span $), r^{2}=0.36$. All relationships significant at $P<0.1$ are shown on the figures.

Multiple regression against SLA and site moisture balance showed that $A_{\text {mass }}, N_{\text {mass }}, A_{\text {area }}$, and $N_{\text {area }}$ were all negatively related to site moisture balance (Table 5). Thus, these measures all tend to be greater in arid than humid environments once variation in SLA is consid- ered. Multiple regression of $A_{\text {area }}$ vs. the combination of $G_{s}$ and site moisture balance showed that $A_{\text {area }}$ tends to be higher in arid environments for a given $G_{s}$, which can be interpreted as a strategy to enhance water conservation. 

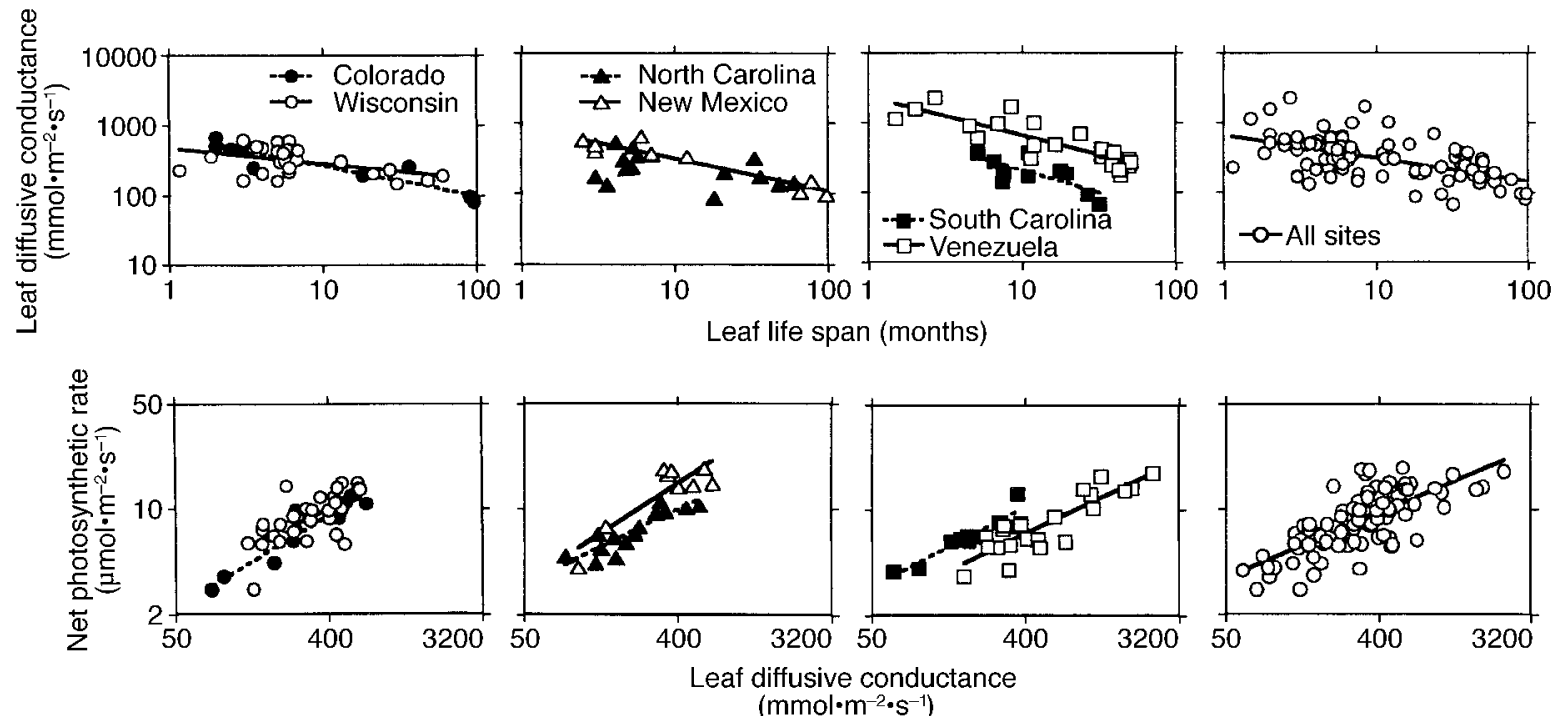

FIG. 2. Leaf diffusive conductance $\left(G_{s}\right)$ in relation to leaf life span (top) and area-based net photosynthetic rate in relation to leaf diffusive conductance (below) for species in six diverse ecosystems. All other details are as in Fig. 1. Correlation coefficients $\left(r^{2}\right)$ for leaf diffusive conductance vs. leaf life span $\left(P<0.001\right.$ unless noted otherwise): CO $\left(r^{2}=0.86\right)$, WI $\left(r^{2}=0.20, P=0.01\right), \mathrm{NC}\left(r^{2}=0.17, P=0.14\right), \mathrm{NM}\left(r^{2}=0.89\right), \mathrm{SC}\left(r^{2}=0.54\right)$, and VEN $\left(r^{2}=0.72\right)$; for $A_{\text {area }}$ vs. leaf diffusive conductance: $\mathrm{CO}\left(r^{2}=0.90\right)$, WI $\left(r^{2}=0.44\right)$, NC $\left(r^{2}=0.77\right)$, NM $\left(r^{2}=0.76\right), \mathrm{SC}\left(r^{2}=0.85\right)$, and VEN $\left(r^{2}=\right.$ $0.75)$. Regression relationships for pooled data: $\log \left(G_{s}\right)=2.82-0.33 \log$ (leaf life span), $r^{2}=0.30 ; \log \left(A_{\text {area }}\right)=-0.28+$ $0.48 \log \left(G_{s}\right), r^{2}=0.54$.

\section{Site differences in mean leaf traits}

Site rankings do not differ in most cases between the average leaf trait data and data standardized by leaf life span. However, site means fail to consider any differences in mean leaf life span across sites (since species were not selected randomly). Mean leaf $N_{\text {mass }}$ did not differ markedly between sites except for South Carolina, where leaf $N_{\text {mass }}$ was lower than other sites (Table 4). Substantial differences in mean SLA were apparent across sites: SLA in desert was far lower than in other sites and was highest in mesic sites. Given that high SLA and leaf $N_{\text {mass }}$ both positively affect $A_{\text {mass }}$ in general (Field and Mooney 1986, Reich et al. 1992, 1994) it is not surprising that N. Carolina (with high mean SLA and $N_{\text {mass }}$ ) had higher mean $A_{\text {mass }}$ than other sites and that New Mexico, Colorado and South Carolina (low SLA and/or low $N_{\text {mass }}$ ) had low $A_{\text {mass }}$ (Table 4). As a consequence of having the "thickest" leaves (low SLA), $N_{\text {area }}$ and $A_{\text {area }}$ were also highest in New Mexico desert-shrubland.

\section{Multivariate analyses}

Simple PCA were performed on all species using five leaf traits (Fig. 6). The first two axes included $90 \%$ of the variation. The positive direction of Axis 1 (the $y$ axis) pointed towards species which, when defined in terms of the leading (and approximately equally important) vectors, were of high SLA, $N_{\text {mass }}, A_{\text {mass, }}$, and short leaf life span. Axis 2 (the $x$ axis) contained only one important vector, $G_{s}$. Coniferous species occupied only the "low SLA" end of Axis 1 and the "low $G_{s}$ ", end of Axis 2, with little overlap with woody pioneers, nonpioneer deciduous trees and shrubs, or herbaceous species. Evergreen broad-leaved species with low leaf turnover rates occupied positions at slightly higher levels on both axes. Although groups do occupy different positions, species within the major groupings were often located at distant positions from one another. Adding the site moisture balance index alone or with the other area-based measures $\left(A_{\text {area }}, N_{\text {area }}\right)$ to the PCA changes the values but not the overall structure, and the climate variable and the area-based leaf traits load onto the second axis, along with $G_{s}$. Hence, the first axis represents variation within and among sites, whereas the second axis largely represents variation among sites, reinforcing the message that climate alters the relationships among leaf traits, largely by altering SLA.

\section{DISCUSSION}

\section{Generality and scaling issues}

The field data from six sites generally support the hypothesis that the slopes of interspecific relationships among leaf traits will be similar among diverse biomes (Reich et al. 1992, Reich 1993) and suggest that the proportional scaling functions between leaf life span, $N_{\text {mass }}$, SLA, $A_{\text {mass }}$, (and to a lesser extent $G_{s}$ and $A_{\text {area }}$ ) across species are general. Thus, for any given proportional difference in one trait among species, the proportional difference among species in any other trait will be similar among all biomes. As an example, for 

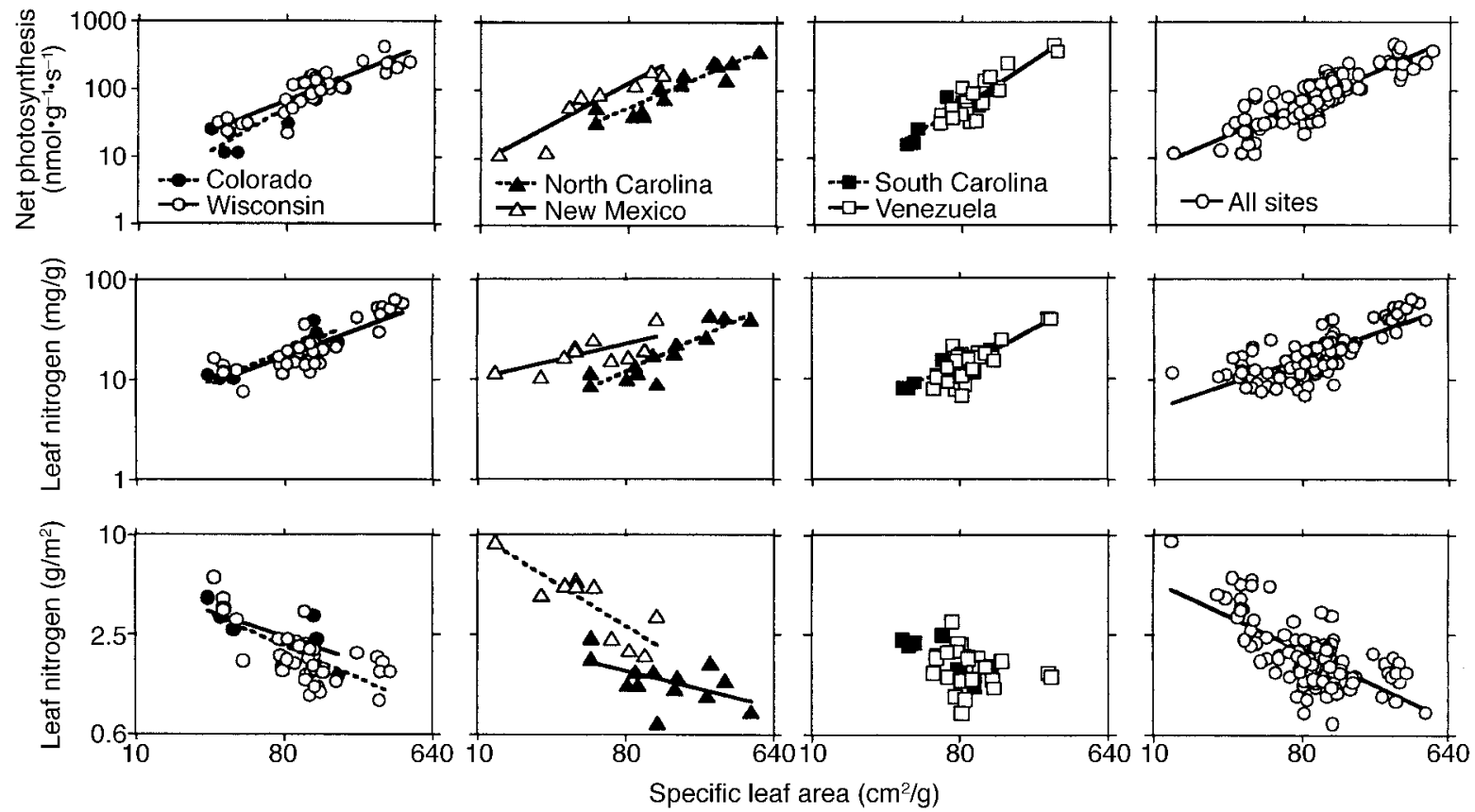

FIG. 3. Mass-based net photosynthetic rates and mass- and area-based leaf $\mathrm{N}$ in relation to specific leaf area for species in six diverse ecosystems. All other details are as in Fig. 1. Correlation coefficients $\left(r^{2}\right)$ for leaf $A_{\text {mass }}$ vs. SLA $(P<0.001$ unless noted otherwise): $\mathrm{CO}\left(r^{2}=0.82\right)$, WI $\left(r^{2}=0.78\right)$, NC $\left(r^{2}=0.86\right)$, NM $\left(r^{2}=0.84\right), \mathrm{SC}\left(r^{2}=0.82\right)$, and VEN $\left(r^{2}\right.$ $=0.79)$; for leaf $N_{\text {mass }}(\mathrm{mg} / \mathrm{g})$ vs. SLA: CO $\left(r^{2}=0.76\right)$, WI $\left(r^{2}=0.62\right)$, NC $\left(r^{2}=0.77\right), \mathrm{NM}\left(r^{2}=0.50\right), \mathrm{SC}\left(r^{2}=0.68\right)$, and VEN $\left(r^{2}=0.64\right)$; and for leaf $N_{\text {area }}\left(\mathrm{g} / \mathrm{m}^{2}\right)$ vs. SLA: CO $\left(r^{2}=0.46\right)$, WI $\left(r^{2}=0.49\right)$, NC $\left(r^{2}=0.30, P=0.05\right)$, NM $\left(r^{2}=0.72\right)$, SC $\left(r^{2}=0.57, P=0.01\right)$, and VEN, NS $)$; Regression relationships for pooled data: $\log \left(A_{\text {mass }}\right)=-0.22+1.08$ $\log (\mathrm{SLA}), r^{2}=0.74 ; \log \left(N_{\text {mass }}\right)=-0.20+0.52 \log (\mathrm{SLA}), r^{2}=0.50 ; \log \left(N_{\text {area }}\right)=1.18-0.47 \log (\mathrm{SLA}), r^{2}=0.45$.

a $10 \times$ decrease in leaf life span in any biome, $A_{\text {mass }}$ will increase by a factor of approximately five.

In contrast to the relatively constant slopes, the elevations of the regression lines often differed by site, with climate-related variation among sites in SLA often driving these differences. Species in an arid desert ecosystem had the lowest SLA on average or at any given leaf N (Fig. 3) or leaf life span (Table 4, Fig. 1), and the most humid sites had the highest average SLA. As a result, the SLA-leaf life span relationship shifts to a higher range of SLA across moist to arid gradients (compare desert with humid montane forest in Fig. 1).

Taken together, the data and analyses from our six sites support the contentions that species, regardless of biome, tend to have a "syndrome" or set of linked leaf traits, with SLA, leaf life span, leaf $N_{\text {mass }}$, and $A_{\text {mass }}$ of each species generally falling together somewhere along a multiple trait continuum (Reich et al. 1991, 1992, 1997). There were no exceptions (outliers): for instance, no species have high $A_{\text {mass }}$ and high SLA, but a long leaf life span and low $N_{\text {mass }}$.

\section{Functional group and phylogenetic considerations}

At all five North American sites, species with high SLA and short leaf life span tended to be herbaceous, with broad-leaved deciduous tree species intermediate in leaf traits and species with low SLA and long leaf life span generally being needle-leaved evergreen conifers. Thus, one could ask whether the observed leaf trait relationships, albeit general across biomes, might be due to comparisons of different plant functional types or evolutionary groupings (in a phylogenetically broad sense). Several contrasts refute that idea and suggest that the trait relationships are general, both within and across broad plant types and taxonomic groups, although different plant groupings do differ in a rough sense (see Fig. 6). First, in the tropical rain forest site, all species were broad-leaved evergreens (i.e., woody Angiosperms), yet the leaf trait relationships in this biome were similar to all other biomes. Second, broadleaved evergreen species in tundra, bog, and forest habitats (e.g., Sarracenia, Kalmia, Lyonia, Quercus virginiana) in Colorado, Wisconsin, and the Carolinas had leaf traits similar to those of tropical rain forest broadleaved evergreen and coniferous needle-leaved species with comparable leaf life spans. Third, needle-leaved deciduous species (Taxodium and Larix) displayed the same collective syndrome of leaf traits as broad-leaved species with similar SLA or leaf life spans, and occupied similar positions along regression lines. These examples suggest that the observed relationships are generally common within or across communities or ecosystems dominated by species of various leaf type (needles, broad) or other functional groups (trees, 

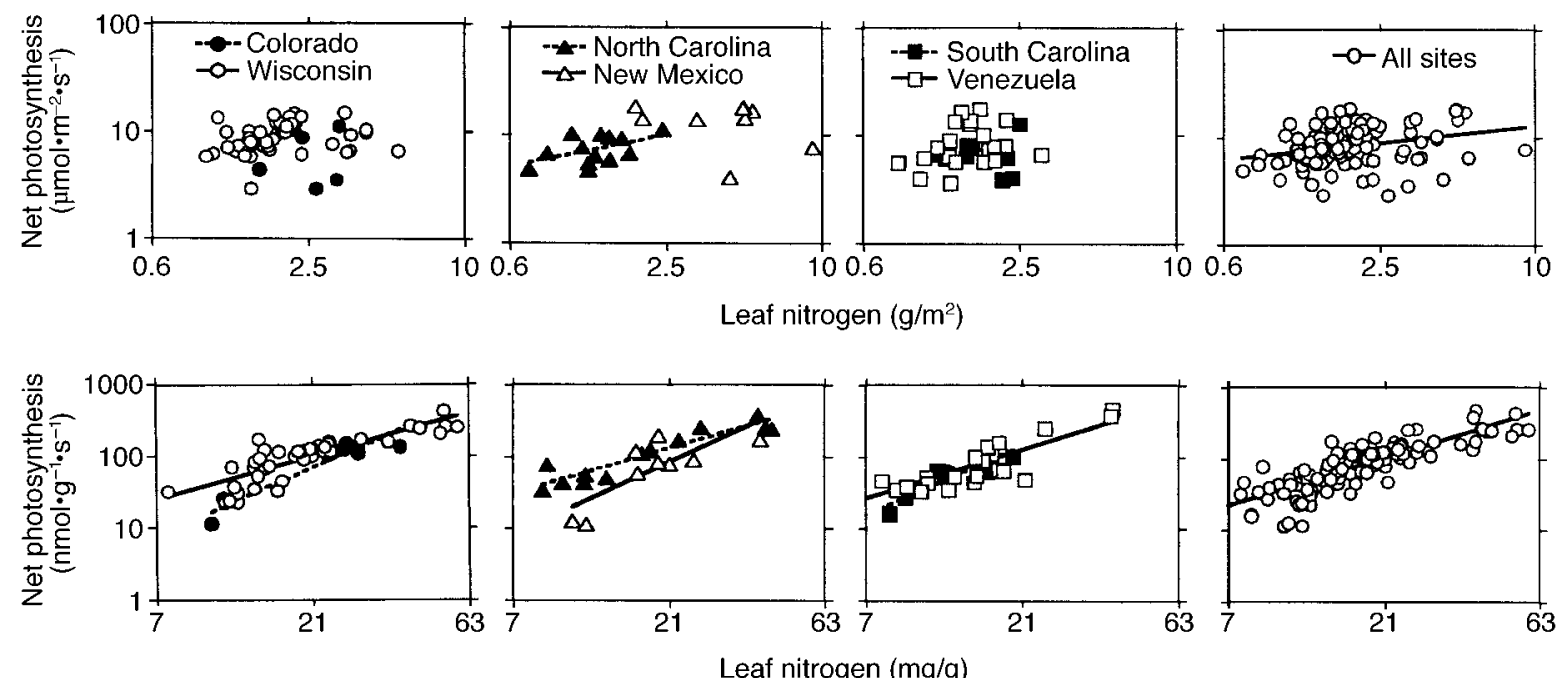

FIG. 4. Area-based net photosynthetic rate $\left(\mu \mathrm{mol} \cdot \mathrm{m}^{-2} \cdot \mathrm{s}^{-1}\right)$ in relation to area-based leaf $\mathrm{N}\left(\mathrm{g} / \mathrm{m}^{2}\right)$, and mass-based net photosynthesis in relation to mass-based leaf $\mathrm{N}(\mathrm{mg} / \mathrm{g})$, for species in six diverse ecosystems. All other details are as in Fig. 1. Correlation coefficients $\left(r^{2}\right)$ for $A_{\text {area }}$ vs. $N_{\text {area }}\left(P<0.001\right.$ unless noted otherwise): CO $\left(r^{2}=0.01, P=0.77\right)$, WI $\left(r^{2}=\right.$ $0.03, P=0.25), \mathrm{NC}\left(r^{2}=0.26, P=0.09\right)$, NM $\left(r^{2}=0.10, P=0.40\right), \mathrm{SC}\left(r^{2}=0.00, P=0.89\right)$, and VEN $\left(r^{2}=0.07, P\right.$ $=0.22)$; for leaf $A_{\text {mass }}$ vs. $N_{\text {mas: }}$ CO $\left(r^{2}=0.90\right)$, WI $\left(r^{2}=0.70\right), \mathrm{NC}\left(r^{2}=0.86\right), \mathrm{NM}\left(r^{2}=0.64\right), \mathrm{SC}\left(r^{2}=0.81\right)$ and VEN $\left(r^{2}=0.70\right)$. Regression relationships for pooled data: $\log \left(A_{\text {area }}\right)=0.85+0.24 \log \left(N_{\text {area }}\right), r^{2}=0.07, P=0.01 ; \log \left(A_{\text {mass }}\right)=$ $0.13+1.42 \log \left(N_{\text {mass }}\right), r^{2}=0.68$.

shrubs, herbs), given the broad variation within each group and the overlap in trait combinations among such groups (Fig. 6, also see Reich et al. 1997). The convergence of these relationships among taxa is also supported using phylogenetically independent contrasts (D. Ackerly and P. Reich, in press).

\section{Controls on photosynthesis}

Similarity in the $A_{\text {mass }}$ to $N_{\text {mass }}$ relationship among species at six sites, as well as in other independent data sets (Field and Mooney 1986, Reich et al. 1992) supports its universal application among all species (but not within species [see Reich et al. 1994]). In contrast,

TABLE 5. Summary of multiple regression analyses for leaf traits in relation to the site moisture balance index (mean annual precipitation minus estimated mean annual pan evaporation) and other leaf traits.

\begin{tabular}{|c|c|c|c|c|c|c|c|c|c|}
\hline \multirow[b]{2}{*}{ Traits $\dagger$} & \multicolumn{2}{|c|}{ Whole model } & \multicolumn{2}{|c|}{ Site moisture balance } & \multicolumn{3}{|c|}{ Independent variable } & \multicolumn{2}{|c|}{ Interaction } \\
\hline & $P$ & $r^{2}$ & $P$ & $F$ & Variable & $P$ & $F$ & $P$ & $F$ \\
\hline$\overline{N_{\text {mass }}}$ & $<0.0001$ & 0.59 & NS & & $\begin{array}{l}\text { leaf life } \\
\text { span }\end{array}$ & $<0.0001$ & 150.6 & NS & \\
\hline$N_{\text {area }}$ & $<0.0001$ & 0.39 & $<0.0001$ & 58.0 & $\begin{array}{l}\text { leaf life } \\
\text { span }\end{array}$ & $<0.0001$ & 17.2 & NS & \\
\hline SLA & $<0.0001$ & 0.75 & $<0.0001$ & 79.4 & $\begin{array}{l}\text { leaf life } \\
\text { span }\end{array}$ & $<0.0001$ & 267.3 & NS & \\
\hline$A_{\text {mass }}$ & $<0.0001$ & 0.83 & $<0.0001$ & 31.2 & $\begin{array}{l}\text { leaf life } \\
\text { span }\end{array}$ & $<0.0001$ & 511.5 & NS & \\
\hline$A_{\text {area }}$ & $<0.0001$ & 0.39 & 0.03 & 5.1 & $\begin{array}{l}\text { leaf life } \\
\text { span }\end{array}$ & $<0.0001$ & 54.5 & NS & \\
\hline$G_{S}$ & $<0.0001$ & 0.39 & $<0.0001$ & 12.8 & $\begin{array}{l}\text { leaf life } \\
\text { span }\end{array}$ & $<0.0001$ & 51.9 & NS & \\
\hline$N_{\text {mass }}$ & $<0.0001$ & 0.64 & 0.002 & 10.6 & SLA & $<0.0001$ & 165.4 & 0.02 & 5.9 \\
\hline$N_{\text {area }}$ & $<0.0001$ & 0.59 & 0.002 & 10.6 & SLA & $<0.0001$ & 69.2 & 0.02 & 5.9 \\
\hline$A_{\text {mass }}$ & $<0.0001$ & 0.78 & $<0.0001$ & 15.6 & SLA & $<0.0001$ & 347.8 & NS & \\
\hline$A_{\text {area }}$ & $<0.0001$ & 0.13 & 0.001 & 12.3 & SLA & 0.006 & 8.1 & NS & \\
\hline$G_{S}$ & $<0.0001$ & 0.12 & NS & & SLA & 0.02 & 6.7 & NS & \\
\hline$A_{\text {mass }}$ & $<0.0001$ & 0.71 & 0.004 & 8.7 & leaf $N_{\text {mass }}$ & $<0.0001$ & 247.0 & NS & \\
\hline$A_{\text {area }}$ & 0.01 & 0.09 & NS & & leaf $N_{\text {area }}$ & NS & & NS & \\
\hline$A_{\text {area }}$ & $<0.0001$ & 0.71 & $<0.0001$ & 55.5 & & $<0.0001$ & 206.3 & NS & \\
\hline
\end{tabular}

Note: If the interaction term was not significant it was dropped from the model.

$\dagger$ Dependent variables 


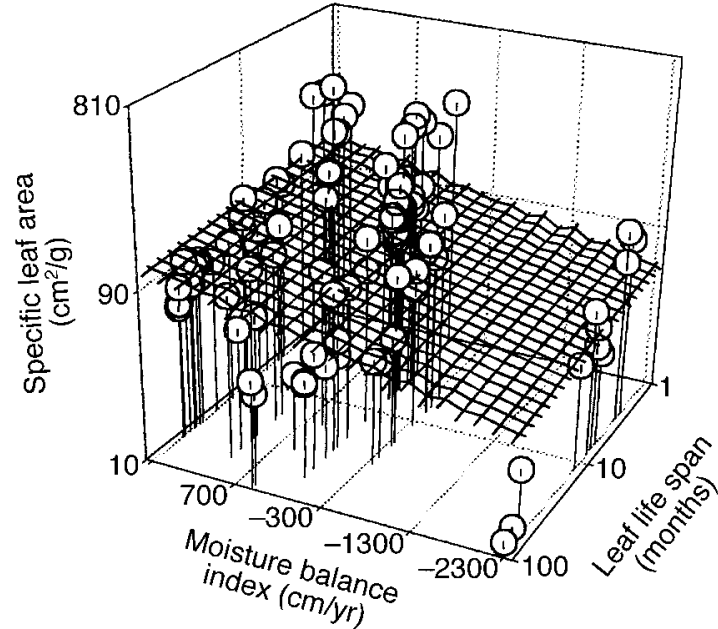

FIG. 5. SLA in relation to the combination of leaf life span and the site moisture balance index (mean annual precipitation minus potential evapotranspiration) for species pooled from six sites in diverse biomes and climates $\left(r^{2}=\right.$ $0.75)$.

relationships between leaf life span, $A_{\text {area }}$, and $N_{\text {area }}$ were more variable. The lack of correlation of leaf $N_{\text {area }}$ and leaf life span is the result of offsetting influences on $N_{\text {area }}$ of SLA and $N_{\text {mass }}$ as they vary with leaf life span: parallel decreases in SLA and $N_{\text {mass }}$ result in minimal or no net change in $N_{\text {area }}$ on average across the leaf lifespan gradient (Fig. 1).

Variation in $A_{\text {area }}$ among species was significantly correlated with leaf $N_{\text {area }}$ in the Field and Mooney
(1986) data survey, but was not related to $N_{\text {area }}$ in any site in this study or in a prior literature survey (Reich et al. 1992). This suggests that there is not a fundamental relationship of $A_{\text {area }}$ to $N_{\text {area }}$ among all species (as discussed previously in Reich et al. 1992, 1994). This may be partially ascribed to offsetting relationships: $A_{\text {mass }}$ is a positive function of $N_{\text {mass }}$ and although $N_{\text {mass }}$ decreases with decreasing SLA, decreasing SLA increases $N_{\text {area }}$ for any given $N_{\text {mass }}$. This results in the potential for leaves to have similar $N_{\text {area }}$ but different $N_{\text {mass }}$. At a given $N_{\text {area }}$, leaves with higher $N_{\text {mass }}$ realize a higher $A_{\text {area }}$ than leaves with lower $N_{\text {mass }}$, due to the positive relationship between $A_{\text {mass }}$ and $N_{\text {mass }}$ (Reich and Walters 1994), giving rise to considerable scatter among species in the relationship of $A_{\text {area }}$ to $N_{\text {area }}$.

Why is the close association of multiple leaf traits general among species and biomes?

To build the physically sturdy foliage common in species with long leaf life span requires proportionally greater carbon than nutrient investment (cell walls, thick waxy cuticles, etc.) and a dense structure. A dense (high mass per volume) or a thick structure corresponds with higher mass per unit area (low SLA) (Abrams et al. 1994, Garnier and Laurent 1994). High C/N ratios and physically tough foliage both likely contribute to the achievement of long leaf life spans (Reich et al. 1991), since they confer some protection from herbivory (Coley 1988) and physical weathering. Lower $N_{\text {mass }}$ is related to lower $A_{\text {mass }}$ because of the central role of $\mathrm{N}$ in photosynthetic enzymes and pigments (Field and Mooney 1986). Thus, lower SLA is associated with

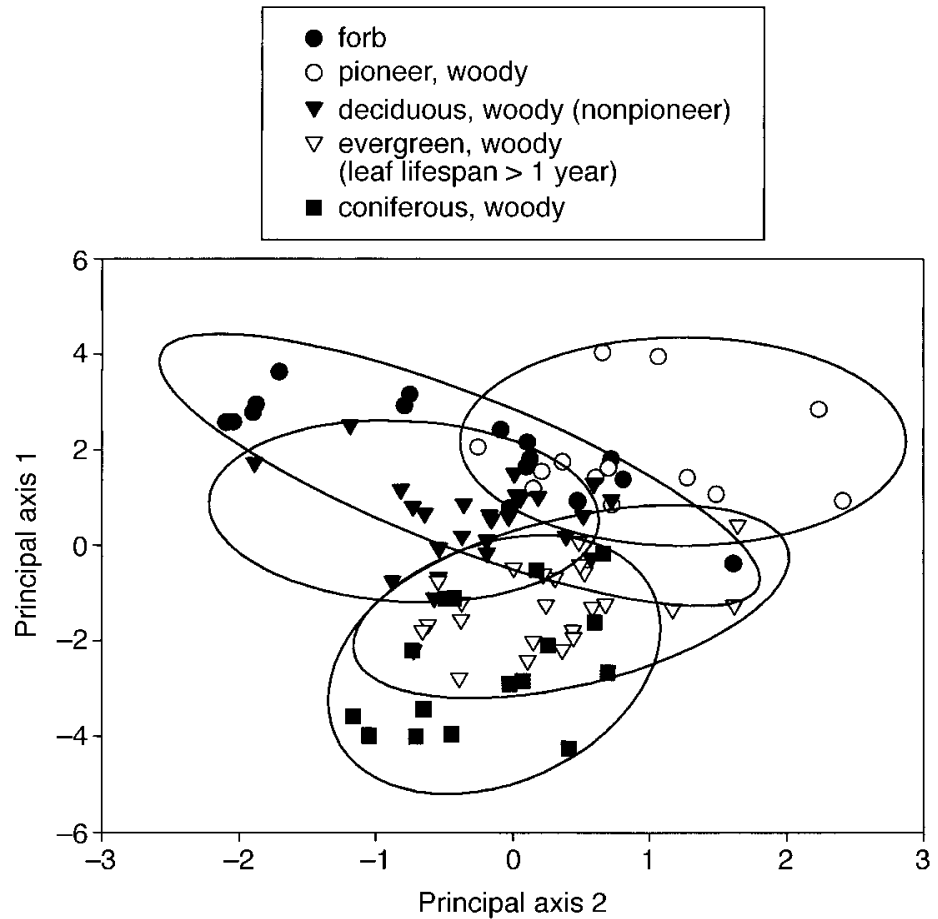

FIG. 6. Principal components analysis of species from six biomes, ordinated according to five leaf traits (mass-based leaf N, mass-based net photosynthetic capacity, leaf life span, specific leaf area, and leaf diffusive conductance, using all 96 species for which data exist for all five traits). The main contributors to high values of the first principal axis were increasing $\mathrm{N}$, photosynthetic capacity, and specific leaf area, and decreasing leaf life span. The main contributor to high values of the second principal axis was leaf diffusive conductance. The encircled groups of points represent five recognizable plant groups. The pioneer, broadleaved deciduous (nonpioneer), and broadleaved evergreen (leaf life span $>1 \mathrm{yr}$ ) groups all include broadleaved woody species and are further separated based on successional habit and phenology. 
greater tissue density and greater allocation of biomass to structural rather than metabolic components, enhancing leaf strength and durability, but also resulting in greater internal shading and potential diffusional limitations (Lloyd et al. 1992, Terashima and Hikosaka 1995, Parkhurst 1994). Thus, low $N_{\text {mass }}$ and low SLA combine to promote low maximum rates of $\mathrm{CO}_{2}$ exchange.

In species with the opposite leaf trait syndrome, the combination of a high $\mathrm{N}$ investment in photosynthetic enzymes and pigments and high SLA can provide high metabolism (gas exchange rates) and light harvesting per unit tissue mass (Field and Mooney 1986, Reich et al. 1992). However, such tissues are also nutritionally desirable to herbivores (Coley et al. 1985) and less well defended physically against biotic (herbivory) and abiotic (physical weathering and stress) agents (Coley et al. 1985, Coley 1988, Reich et al. 1991).

Given the different roles of $\mathrm{C}$ vs. $\mathrm{N}$ in leaves, and of $\mathrm{C}$ or $\mathrm{N}$ allocated to structural vs. metabolic functions, it may be physically impossible to construct a leaf with very high $N_{\text {mass }}$ and net $\mathrm{CO}_{2}$ exchange characteristics that is also physically robust and durable. Such constraints likely place a limit on how far any species can occur (Fig. 7a) above the regression lines shown in Fig. 1-4. In contrast, although biophysically feasible to build, no species has flimsy, unproductive leaves; there would be disadvantages to leaves that are neither persistent nor productive and such a leaf trait syndrome would likely be maladaptive. Thus, selection should place a limit on how far any species can be (Fig. 7a) below the regression lines shown in Fig. 1-4. Given these constraints, the "solution" for every species is a position on the continuum of the leaf trait syndrome, ranging from species with leaves weighted toward productivity, others with leaves weighted toward persistence, and others at points in between.

\section{Biogeographic differences}

Do species in cold climates have high leaf $N_{\text {mass }}$ ? Leaf $N_{\text {mass }}$ did not differ among sites except that it was lower on infertile sandy soils in subtropical forest than all other sites. Thus the data presented offer no evidence of higher leaf $N_{\text {mass }}$ in high elevation or cold-climate sites, superficially disagreeing with Körner (1989). However, site differences in soils could mask any temperature trend for $N_{\text {mass }}$, given large variation in soil fertility and a low sample size ( $n=6$ sites). Moreover, other evidence does suggest that within phylogenetically similar groups, plants adapted to colder environments may have higher $N_{\text {mass }}$ (Körner 1989, Reich et al. 1996).

Our data provide mixed support for the idea that desert plants are rich in N. On average, we found no evidence of higher average $N_{\text {mass }}$ in desert plants, disagreeing with long-mentioned hypotheses, but agreeing with the recent analysis by Killingbeck and Whitford (1996). However, due to low SLA, $N_{\text {area }}$ was higher in

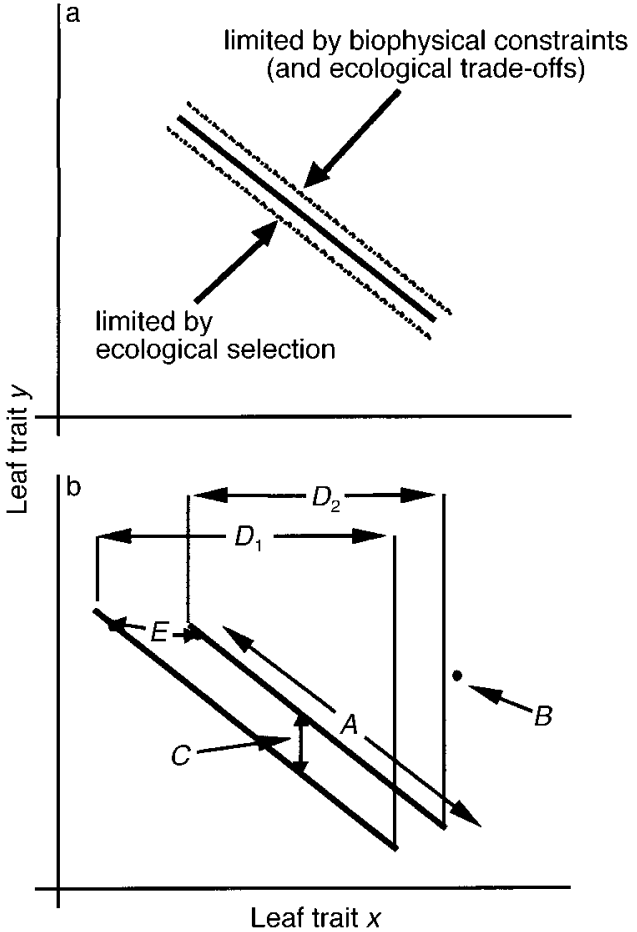

FIG. 7. (a) Hypothetical limitations to leaf trait relationships due to biophysical constraints, physiology, ecology, and natural selection. (b) Conceptualization of implications of leaf trait relationships for intra- and interecosystem comparisons. $A$ shows typical variation within a site, $B$ illustrates a hypothetical exception ("outlier") species, $C$ illustrates leaf trait $y$ in two different ecosystems at a common leaf trait $x$, $D$ illustrates differences in the range of leaf trait $x$ in two different ecosystems, and $E$ illustrates two species in different ecosystems that occupy the same relative intra-ecosystem ranking.

desert-shrub vegetation than elsewhere, both on average and for any given leaf life span. At any given SLA, moreover, $N_{\text {area }}, N_{\text {mass }}$, and $A_{\text {mass }}$ were higher in desert shrub vegetation than in other biomes (Table 3 ) and higher as a function of increasing site moisture deficit (Table 5). Thus, leaves of desert plants are N-rich compared to all others when comparing leaves of comparable structure. The high $\mathrm{N}$ of desert plants at any given SLA offsets the negative correlation between $N_{\text {mass }}$ and SLA and the tendency for desert plants to have low SLA, resulting in desert plants having similar average $N_{\text {mass }}$ as in other biomes.

Variation in SLA was related to site microenvironment (see Fig. 5) and large differences in SLA occur across sites for any given leaf life span. These patterns are consistent with long-held common knowledge about variation in SLA, but to our knowledge, we are unaware of any published approach to quantifying these differences.

\section{Implications}

These comparisons of leaf trait relationships in widely disparate ecosystems and biomes have several im- 
plications. First, they provide a quantitative basis for evaluating intra-ecosystem species differences ( $A$, Fig. $7 b)$ and for identifying exceptions that might occur due to unique plant adaptations ( $B$, Fig. $7 b$ ). Second, identification of general leaf scaling relationships enables contrasts among ecosystems and biomes, and serves as a means of quantifying differences among them. For example, if species in a given ecosystem have higher or lower leaf $\mathrm{N}$ at any given SLA or leaf life span than in another ecosystem ( $C$, Fig. $7 b$ ), or if the range of leaf $\mathrm{N}$, leaf life span, or SLA is different in one ecosystem compared to another ( $D$, Fig. $7 \mathrm{~b})$, then this is likely due to key and quantifiable differences in these ecosystems (e.g., climate, soils, biology). Third, these scaling relationships allow quantitative comparisons of species in widely differing ecosystems. For example, do species (from different ecosystems) that share the same relative intra-ecosystem ranking in leaf traits share the same successional position ( $E$, Fig. 7b)? Existing data suggest that such patterns often hold. For example, tree species of high SLA, high leaf $N_{\text {mass }}$, and short leaf life span are more likely to be early successional, inhabit high light microsites and display a fast growth rate in both temperate and tropical ecosystems (Uhl 1987, Reich et al. 1991, 1992). Finally, the uncovering of the generality of leaf trait relationships should allow the selection of useful foliage attributes for modeling vegetation productivity, distribution, and dynamics at diverse levels of scale (Running and Hunt 1993, Leuning et al. 1995, Aber et al. 1996).

\section{ACKNOWLEDGMENTS}

This research was partially supported by National Science Foundation Grants BSR 8819718, BSR 8857129, and IBN9296005, and by the NSF Long-Term Ecological Research Program. D. S. Ellsworth was supported by the U.S Department of Energy (DE-ACO2-76CH00016) during the preparation of this manuscript. We thank Chris Uhl, Brian Kloeppel, Dan Bahauddin, and many others who contributed to this work in a wide variety of ways. We thank A. Thomas and Barbara G. Hallowell for providing unpublished data on leaf longevity in Galax.

\section{Literature Cited}

Aber, J. D., P. B. Reich, and M. L. Goulden. 1996. Extrapolating leaf $\mathrm{CO}_{2}$ exchange to the canopy: a generalized model of forest photosynthesis validated by eddy correlation. Oecologia 106:267-275.

Abrams, M. D., M. E. Kubiske, and S. A. Mostoller. 1994. Relating wet and dry year ecophysiology to leaf structure in contrasting temperate tree species. Ecology 75:123-133.

Ackerly, D., and P. B. Reich. 1999. Convergence and correlations among leaf physiological traits: a test using independent contrasts. American Journal of Botany, in press.

Beadle, N. C. 1966. Soil phosphate and its role in molding segments of the Australian flora and vegetation, with special reference to xeromorphy and scleromorphy. Ecology 47:992-1007.

Chabot, B. F., and D. J. Hicks. 1982. The ecology of leaf life spans. Annual Review of Ecology and Systematics 13 229-259.

Chazdon, R. L., and C. B. Field. 1987. Determinants of photosynthetic capacity in six rainforest Piper species. Oecologia 73:222-230.
Coley, P. D., J. P. Bryant, and F. S. Chapin III. 1985. Resource availability and plant anti-herbivore defense. Science $\mathbf{2 3 0}$ : 895-899.

Coley, P. D. 1988. Effects of plant growth rate and leaf lifetime on the amount and type of anti-herbivore defense. Oecologia 74:531-536.

Ellsworth, D. S., and P. B. Reich. 1992. Leaf mass per area, nitrogen content and photosynthetic carbon gain in Acer saccharum seedlings in contrasting forest light environments. Functional Ecology 6:423-435.

Field, C., and H. A. Mooney. 1986. The photosynthesisnitrogen relationship in wild plants. Pages $25-55$ in T. Givnish, editor. On the economy of plant form and function. Cambridge University Press, London, UK.

Garnier, E., and G. Laurent. 1994. Leaf anatomy, specific mass and water content in congeneric annual and perennial grass species. New Phytologist 128:725-736.

Killingbeck, K. T., and W. G. Whitford. 1996. High foliar nitrogen in desert shrubs: an important ecosystem trait or defective desert doctrine? Ecology 77:1728-1737.

Körner, Ch. 1989. The nutritional status of plants from high altitudes. A worldwide comparison. Oecologia 81:379391.

Leuning, R., F. M. Kelliher, D. G. G. de Prury, and E.-D. Schulze. 1995. Leaf nitrogen, photosynthesis, conductance and transpiration: scaling from leaves to canopy. Plant, Cell and Environment 18:1183-1200.

Lugg, D. G., and T. R. Sinclair. 1981. Seasonal changes in photosynthesis of field-grown soybean leaflets. 2. Relation to nitrogen content. Photosynthetica 15:138-144.

Lloyd, J., J. P. Syvertsen, P. E. Kriedemann, and G. D. Farquhar. 1992. Low conductances for $\mathrm{CO}_{2}$ diffusion from stomata to the sites of carboxylation in leaves of woody species. Plant, Cell and Environment 15:873-899.

Mulkey, S. S., K. Kitajima, and S. J. Wright. 1995. Photosynthetic capacity and leaf longevity in the canopy of a dry tropical forest. Selbyana 16:169-173.

Parkhurst, D. F. 1994. Diffusion of $\mathrm{CO}_{2}$ and other gases inside leaves. New Phytologist 126:449-479.

Reich, P. B. 1993. Reconciling apparent discrepancies among studies relating life span, structure and function of leaves in contrasting plant life forms and climates: "the blind men and the elephant retold." Functional Ecology 7:721-725.

Reich, P. B., J. Oleksyn, and M. G. Tjoelker. 1996. Needle respiration and nitrogen concentration in Scots pine populations from a broad latitudinal range: a common garden test with field grown trees. Functional Ecology 10:768776.

Reich, P. B., C. Uhl, M. B. Walters, and D. S. Ellsworth. 1991. Leaf lifespan as a determinant of leaf structure and function among 23 tree species in Amazonian forest communities. Oecologia 86:16-24.

Reich, P. B., and M. B. Walters. 1994. Photosynthesis-nitrogen relations in Amazonian tree species. II. Variation in nitrogen vis-a-vis specific leaf area influences mass and area-based expressions. Oecologia 97:73-81.

Reich, P. B., M. B. Walters, and D. S. Ellsworth. 1992. Leaf lifespan in relation to leaf, plant and stand characteristics among diverse ecosystems. Ecological Monographs 62: 365-392.

Reich, P. B., M. B. Walters, and D. S. Ellsworth. 1997. From tropics to tundra: global convergence in plant functioning. Proceedings of the National Academy of Sciences, USA 94:13730-13734.

Reich, P. B., M. B. Walters, D. S. Ellsworth, and C. Uhl. 1994. Photosynthesis-nitrogen relations in Amazonian tree species. I. Patterns among species and communities. Oecologia 97:62-72.

Running, S. W., and E. R. Hunt. 1993. Generalization of a forest ecosystem process model for other biomes, BIOME- 
BGC, and an application for global-scale models. Pages 141-158 in Ehleringer, J. R., and C. B. Field, editors. Scaling physiological processes: leaf to globe. Academic Press, San Diego, California, USA.

Sokal, R. R., and F. J. Rohlf. 1995. Biometry. Third edition. W. H. Freeman, New York, New York, USA.

Steel, R. G. D., and J. H. Torrie. 1980. Principles and procedures of statistics. A biometrical approach. Second edition. McGraw-Hill, New York, New York, USA.
Terashima, I., and K. Hikosaka. 1995. Comparative ecophysiology of leaf and canopy photosynthesis. Plant, Cell and Environment 18:1111-1128.

Uhl, C. 1987. Factors controlling succession following slashand-burn agriculture in Amazonia. Journal of Ecology 75: 377-407.

Williams, K., C. B. Field, and H. A. Mooney. 1989. Relationships among leaf construction cost, leaf longevity, and light environment in rain forest plants of the genus Piper. American Naturalist 133:198-211. 